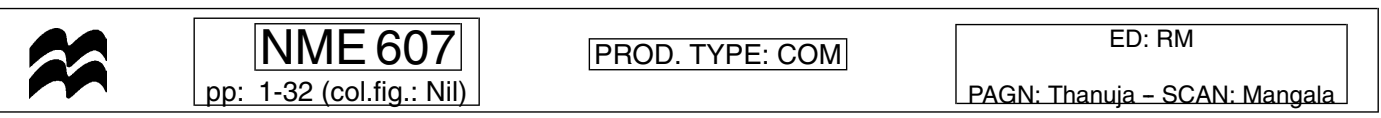

INTERNATIONAL JOURNAL FOR NUMERICAL METHODS IN ENGINEERING

Int. J. Numer. Meth. Engng 2003; 56:000-000 (DOI: 10.1002/nme.607)

\title{
On the strong discontinuity approach in finite deformation settings
}

\author{
J. Oliver ${ }^{* \dagger}$, A. E. Huespe ${ }^{\ddagger \S}$, M. D. G. Pulido and E. Samaniego \\ E.T.S. Enginyers de Camins, Canals i Ports, Technical University of Catalonia, Campus Nord UPC, \\ Mòdul Cl, Gran Capitán s/n, 08034 Barcelona, Spain
}

SUMMARY

Taking the strong discontinuity approach as a framework for modelling displacement discontinuities and strain localization phenomena, this work extends previous results in infinitesimal strain settings to finite deformation scenarios.

11 By means of the strong discontinuity analysis, and taking isotropic damage models as target continuum (stress-strain) constitutive equation, projected discrete (tractions-displacement jumps) constitutive

13 models are derived, together with the strong discontinuity conditions that restrict the stress states at the discontinuous regime. A variable bandwidth model, to automatically induce those strong discontinuity

15 conditions, and a discontinuous bifurcation procedure, to determine the initiation and propagation of the discontinuity, are briefly sketched. The large strain counterpart of a non-symmetric finite element

17 with embedded discontinuities, frequently considered in the strong discontinuity approach for infinitesimal strains, is then presented. Finally, some numerical experiments display the theoretical issues, and

19 emphasize the role of the large strain kinematics in the obtained results. Copyright (c) 2003 John Wiley \& Sons, Ltd.

21 KEY WORDS: strong discontinuities; localization; fracture; damage; finite strains

\section{INTRODUCTION}

23 Modelling the onset and development of material discontinuities (fractures, cracks, slip lines, etc.) has been the object of intense research in solid mechanics during the last decades.

25 Besides the classical non-linear fracture mechanics approaches [1], one common way of modelling displacement discontinuities, from the continuum mechanics point of view, has been

\footnotetext{
${ }^{*}$ Correspondence to: J. Oliver, E.T.S. Enginyers de Camins, Canals i Ports, Technical University of Catalonia, Campus Nord UPC, Mòdul C1, Gran Capitán s/n, 08034 Barcelona, Spain

†E-mail: oliver@cimne.upc.es

‡E-mail: ahuespe@intec.unl.edu.ar

$\S$ CIMEC/CONICET-UNL, Argentina
}

Contract/grant sponsor: Spanish Ministry of Science and Technology; contract/grant number: MAT-2001-3863-C03-03. Contract/grant sponsor: Secretaria de Estado de Educación

Received 28 June 2001

Copyright (c) 2003 John Wiley \& Sons, Ltd. 


\section{NME 607}

1 the simulation of the strain localization phenomenon by resorting to material models equipped with strain-softening. This can be justified not only from the physical point of view, since this

3 mode of deformation can be observed either in ductile materials (see, for example, Reference [2] and references therein and Reference [3]) or in quasibrittle materials [4], but also from

5 the kinematic point of view, since strain localization induces relative displacements at both side of the localization band that can be interpreted as displacement jumps. However, it is nowadays well known that classical continuum inviscid dissipative models featuring strain softening lead to ill-posed boundary value problems. This becomes particularly evident in

9 numerical simulation contexts since the obtained finite element results exhibit strong mesh dependence and no convergence with mesh refinement.

11 Different remedies for this behaviour have been presented in the literature. Basically, they are based on the modification of the classical inviscid constitutive response, by adding, to the

13 stress-strain constitutive equation, higher-order deformation gradients, non-local dependence or rate dependence [5].

15 In recent years, a second group of procedures that resort to the strong discontinuity concept have been developed. They advocate the introduction of the strong discontinuity kinematics,

17 i.e. the modification of the standard continuum kinematical descriptions to take into account the appearance of discontinuous displacement fields through material interfaces in the solid

19 [6-11]. A common issue associated to these procedures is the finite element technology, which should enable to capture jumps in the displacement field. For such purposes, new families of

21 elements with embedded discontinuities have been developed [12-16].

Considering the aforementioned strong discontinuity kinematics has some interesting con-

23 sequences. In fact, it turns out [17] that under such a kinematics standard continuum (stressstrain) constitutive models induce discrete (traction-displacement jump) constitutive models

25 on the interface of discontinuity. Those discrete models can then be regarded as projections of the original constitutive model on that discontinuity interface, and inherit the basic features

27 of the parent continuum model $[17,18]$. However, they can be only induced when a particular stress state has been reached at the interface, which is therefore restricted by the so-called

29 strong discontinuity conditions [17].

Consequently, and regarding the way that different models make use of those induced

31 discrete models, and the format in which they are introduced into the analysis, they can be classified into:

33 1. Discrete approaches $[11-13,19]$ : They introduce a discrete constitutive model at the interface that is completely independent from the continuum one. Their connection with

35 the strong discontinuity kinematics lies in numerical aspects, essentially in the use of finite elements with embedded discontinuities.

37 2. Discrete-continuum approaches $[8,10,20,21]$ : They make use of the continuum induced discrete constitutive equation introducing it into the problem in a discrete format: i.e. the

39 discrete constitutive equation is analytically derived and then introduced, as a separation law, at the discontinuous interface regardless the fulfillment of the strong discontinuity conditions.

\footnotetext{
IA crucial condition for this to happen is that the strong discontinuity kinematics is linked to the continuum constitutive model through a constitutive regularization of the hardening/softening parameter. This allows the model to return bounded tractions for input unbounded strains.
} 


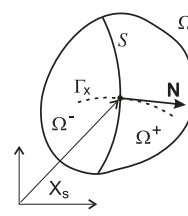

(a)
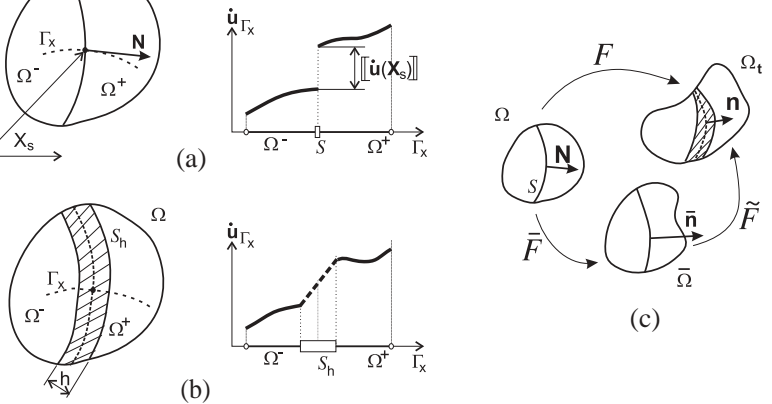

(b)

(c)

Figure 1. (a) Strong discontinuity kinematics; (b) regularized kinematics; and

(c) multiplicative decomposition.

3. Continuum approaches: A full use of the connections between the continuum and the induced discrete constitutive models is made. As a matter of fact the latter is never explicitly introduced at the discontinuous interface, but it is implicitly induced from the former as a consequence of the activation of the strong discontinuity kinematics once the strong discontinuity conditions are fulfilled. As a result, the whole analysis and simulation is kept in the continuum format.

7 This paper focuses on this last continuum approach that, from now on, will be termed the strong discontinuity approach (SDA). Its analysis and implications for infinitesimal strains

9 settings have been analysed by the authors in the past [7, 14, 17, 18, 22-25], and here we extend them to the finite deformation setting.

11 The aim of this work is then to explore the requirements and consequences of using a full continuum approach for modelling strong discontinuities in the large strain scenario. It is not

13 intended to make a comparative study between the aforementioned approaches or to state the possible benefits of the SDA in comparison with other modelling tools, but to show that the

15 SDA methodology and concepts, previously developed for the infinitesimal strain case, can be generalized to finite strains.

17 The remaining of this paper is organized as follows: Section 2 introduces the strong discontinuity kinematics in the large strain context. Then, in Section 3, a strong discontinuity

19 analysis is done for an isotropic continuum damage model and the induced discrete constitutive model and the corresponding strong discontinuity conditions are derived. In Section 4 a

21 description of the finite element technology, for the large strain kinematics case, is provided. Section 5 is devoted to present a set of numerical simulations in the context of the SDA.

23 Finally some concluding remarks close the work.

\section{STRONG DISCONTINUITY KINEMATICS}

25 Let $\Omega \in \mathbb{R}^{3}$ be a body undergoing a mechanical process which displays a displacement field that is discontinuous across a material surface $\mathscr{S} \subset \Omega$ (see Figure 1(a)) with a jump in the

27 velocity field given by $\llbracket \dot{\mathbf{u}} \rrbracket=\dot{\mathbf{u}}\left(\mathbf{X}_{\mathscr{S}^{+}}\right)-\dot{\mathbf{u}}\left(\mathbf{X}_{\mathscr{S}^{-}}\right)$. The velocity field at the material point $\mathbf{X}$ at 
1 time $t$ is described by

$$
\dot{\mathbf{u}}(\mathbf{X}, t)=\dot{\overline{\mathbf{u}}}(\mathbf{X}, t)+\mathscr{H}_{s} \llbracket \dot{\mathbf{u}} \rrbracket(\mathbf{X}, t) ; \quad \mathscr{H}_{s}(\mathbf{X})=\left\{\begin{array}{cc}
0 & \forall \mathbf{X} \in \Omega^{-} \\
1 & \forall \mathbf{X} \in \Omega^{+}
\end{array}\right.
$$

$3 \dot{\overline{\mathbf{u}}}(\mathbf{X})$ and $\llbracket \dot{\mathbf{u}} \rrbracket$ being two continuous fields, $\mathscr{H}_{S}$ is the step function (Heaviside function) and $\Omega^{-}$, $\Omega^{+}$each one of the body's disjunct parts of $\Omega$ obtained from its division by the surface $\mathscr{S}$.

5 This mode is characterized by a material velocity gradient $\dot{\mathbf{F}}$ :

$$
\dot{\mathbf{F}}=\dot{\mathbf{u}} \otimes \nabla=\dot{\overline{\mathbf{F}}}+\delta_{\mathscr{S}}(\llbracket \dot{\mathbf{u}} \rrbracket \otimes \mathbf{N})
$$

7 where $\dot{\overline{\mathbf{F}}}$ is a bounded (regular) term, $\delta_{\mathscr{S}}$ the Dirac's delta function on $\mathscr{S}$, and $\mathbf{N}$ a material (fixed) unit vector orthogonal to $\mathscr{S}$. The deformation gradient $\mathbf{F}(\mathbf{X}, t)$, at time $t$, comes from 9 the integration of Equation (2) along time:

$$
\mathbf{F}(\mathbf{X}, t)=\mathbb{1} \int_{0}^{t} \dot{\overline{\mathbf{F}}} \mathrm{d} t+\int_{t_{\mathrm{SD}}}^{t} \delta \mathscr{S}(\llbracket \dot{\mathbf{u}} \rrbracket \otimes \mathbf{N}) \mathrm{d} t=\underbrace{\overline{\mathbf{F}}}_{\text {bounded }}+\delta \mathscr{S}(\boldsymbol{\beta} \otimes \mathbf{N})
$$

11 where $t_{\mathrm{SD}}(\mathbf{X})$ stands for the onset time of the strong discontinuity mode at the material point $\mathbf{X}$, and $\boldsymbol{\beta}(\mathbf{X}, t)$ is the incremental displacement jump between the current time, $t$, and $t_{\mathrm{SD}}$ :

$$
\begin{array}{ll}
\boldsymbol{\beta}=\mathbf{0} ; & t<t_{\mathrm{SD}} \\
\boldsymbol{\beta}=\llbracket \mathbf{u} \rrbracket_{t}-\llbracket \mathbf{u} \rrbracket_{t_{\mathrm{SD}}} ; & t \geqslant t_{\mathrm{SD}}
\end{array}
$$

where $\llbracket \mathbf{u} \rrbracket_{t_{S \mathrm{D}}}$ stands for the apparent displacement jump at the end of the weak discontinuity

15 regime $\left(t=t_{\mathrm{SD}}\right)$ described in Section 3.2. Notice that in Equation (3), the regular term $\overline{\mathbf{F}}$ remains bounded during all the process.

\section{2.1. Multiplicative decomposition of the deformation gradient}

For the subsequent analysis it is convenient to adopt, from Equation (3), the multiplicative

19 decomposition of the deformation gradient (see Figure 1(c)) proposed in Reference [8]:

$$
\mathbf{F}=\tilde{\mathbf{F}} \cdot \overline{\mathbf{F}}=\left(\mathbb{1}+\delta_{\mathscr{S}}(\boldsymbol{\beta} \otimes \overline{\mathbf{n}})\right) \cdot \overline{\mathbf{F}} ; \quad \overline{\mathbf{n}}=\overline{\mathbf{F}}^{-T} \cdot \mathbf{N}
$$

21 which introduces the concept of a regular intermediate configuration $\bar{\Omega}$, described by a $\mathbb{R}^{3}$ mapping whose gradient of deformation is regular and given by $\overline{\mathbf{F}}$. Notice that, in accordance

23 with Equation (5) $\overline{\mathbf{n}}$, the normal vector to the surface $\mathscr{S}$ convected by $\overline{\mathbf{F}} \neq \mathbb{1}$, is not a unit vector.

25 For the sake of simplicity in the subsequent mathematical analysis, we shall regularize the Dirac's delta function by defining a slice of the body $\mathscr{S}_{h}$ (see Figure 1(b)), of finite thickness

$27 h$, which contains the surface $\mathscr{S}\left(\mathscr{S} \subset \mathscr{S}_{h}\right)$. Then we consider the $h$-sequence of regular functions:

$$
\delta_{\mathscr{S}}^{h}=\frac{\mu_{\mathscr{S}}}{h} ; \quad \mu_{\mathscr{S}}= \begin{cases}0 & \forall \mathbf{X} \notin \mathscr{S}_{h} \\ 1 & \forall \mathbf{X} \in \mathscr{S}_{h}\end{cases}
$$

so that, in the limit, as $h \rightarrow 0$ then $\delta_{\mathscr{S}_{h}} \rightarrow \delta_{\mathscr{S}}$. 
1 Using this regularization, and after some algebraic manipulation, the following identities are obtained:

$$
\begin{aligned}
\dot{\mathbf{F}}^{h} & =\dot{\overline{\mathbf{F}}}+\frac{\mu_{\mathscr{S}}}{h}(\dot{\boldsymbol{\beta}} \otimes \mathbf{N}) \\
\mathbf{F}^{h} & =\overline{\mathbf{F}}+\frac{\mu_{\mathscr{S}}}{h}(\boldsymbol{\beta} \otimes \mathbf{N})=\underbrace{\left(\mathbb{1}+\frac{\mu_{\mathscr{S}}}{h}(\boldsymbol{\beta} \otimes \overline{\mathbf{n}})\right)}_{\mathbf{F}^{h}} \cdot \overline{\mathbf{F}}=\tilde{\mathbf{F}}^{h} \cdot \overline{\mathbf{F}} \\
\mathbf{F}^{h^{-1}} & =\overline{\mathbf{F}}^{-1} \cdot\left(\tilde{\mathbf{F}}^{h}\right)^{-1}=\overline{\mathbf{F}}^{-1} \cdot\left(\mathbb{1}-\frac{\mu_{\mathscr{S}}}{h+\boldsymbol{\beta} \cdot \overline{\mathbf{n}}}(\boldsymbol{\beta} \otimes \overline{\mathbf{n}})\right) \\
J^{h} & =\operatorname{det}\left(\mathbf{F}^{h}\right)=\operatorname{det}(\overline{\mathbf{F}})\left(1+\frac{\mu_{\mathscr{S}} \boldsymbol{\beta} \cdot \overline{\mathbf{n}}}{h}\right)=\bar{J} \tilde{J}^{h} \\
\bar{J} & =\operatorname{det}(\overline{\mathbf{F}}) ; \quad \tilde{J}^{h}=\operatorname{det}\left(\tilde{\mathbf{F}}^{h}\right)=\left(1+\frac{\mu_{\mathscr{S}} \boldsymbol{\beta} \cdot \overline{\mathbf{n}}}{h}\right)
\end{aligned}
$$

3 We also define $\mathbf{n}$ as the normal vector $\mathbf{N}$ convected by the total motion,

$$
\mathbf{n}=\mathbf{F}^{-\mathrm{T}} \cdot \mathbf{N}=\tilde{\mathbf{F}}^{h-T} \cdot \overline{\mathbf{n}}=\frac{\overline{\mathbf{n}}}{\tilde{J}^{h}}
$$

5 where Equations (8), (11) and (5) have been used."

\section{STRONG DISCONTINUITY ANALYSIS}

7 In addition to the kinematics described in previous sections, the SDA lies on several assumptions and ingredients, some of them trying to match the physical aspects associated to

9 the formation of a displacement discontinuity and some others of more mathematical nature. Those assumptions and their implications will be described in the following sections.

\section{3.1. Traction continuity: stress boundedness}

Let us consider the material configuration of the solid, $\Omega$, with boundary $\partial \Omega=\Gamma_{u} \cup \Gamma_{\sigma}$, where

$13 \Gamma_{u}$ is the part of that boundary where displacements are prescribed and $\Gamma_{\sigma}$ the one were tractions are given (see Figure 2), crossed by the discontinuity interface $\mathscr{S}$ that splits $\Omega$ into

15 the domains $\Omega^{+}$and $\Omega^{-}$. The local equilibrium of the body is described by the following equations:

17

$$
\begin{array}{ll}
\mathbf{P} \cdot \nabla_{\mathbf{X}}+\boldsymbol{\rho}_{0} \overline{\mathbf{B}}=\mathbf{0} & \text { for }(\mathbf{X}, t) \in \Omega \backslash \mathscr{S} \times[0, T] \\
\mathbf{P} \cdot \mathbf{N}=\mathscr{T}^{\mathrm{ext}} & \text { for }(\mathbf{X}, t) \in \Gamma_{\sigma} \times[0, T] \\
\mathbf{P}_{\Omega^{+}} \cdot \mathbf{N}=\mathbf{P}_{\Omega^{-}} \cdot \mathbf{N} & \text { for }(\mathbf{X}, t) \in \mathscr{S} \times[0, T]
\end{array}
$$

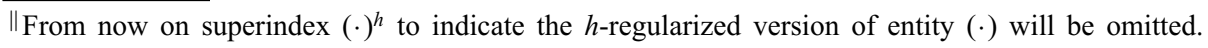




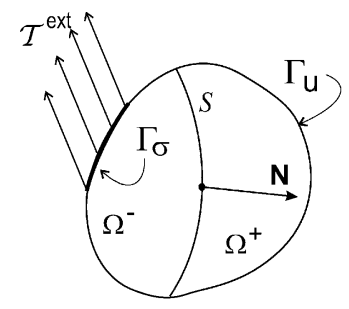

Figure 2. Strong discontinuity in a body.

1 where $\mathbf{P}(\mathbf{X}, t)$ is the nominal (first Piola-Kirchhoff) stress tensor $\left(\mathbf{P}_{\Omega^{+}}\right.$and $\mathbf{P}_{\Omega^{-}}$being its values at the domains $\Omega^{+}$and $\Omega^{-}$, respectively), $\boldsymbol{\rho}_{0}(\mathbf{X})$ is the density, $\overline{\mathbf{B}}(\mathrm{X}, t)$ are the body

3 forces, $\mathscr{T}^{\operatorname{ext}}(\mathbf{X}, t)$ stands for the external forces applied at the boundary $\Gamma_{\sigma}$ and $[0, T]$ is the time interval of interest.

5 In the context of the SDA and the regularized kinematics of Section 2.1 we extend, as an 'ad hoc' hypothesis, the traction continuity equation (13c) to the interior of the discontinuity

7 interface $\mathscr{S}_{h}$ of Figure 1(b):

$$
\mathscr{T}=\mathbf{P}_{\Omega^{+}} \cdot \mathbf{N}=\mathbf{P}_{\Omega^{-}} \cdot \mathbf{N}=\mathbf{P}_{\mathscr{S}} \cdot \mathbf{N}
$$

9 where $\mathscr{T}$ stands for the nominal traction vector and $\mathbf{P}_{\mathscr{S}}=\left.\mathbf{P}(\mathbf{X}, t)\right|_{\mathbf{X} \in \mathscr{S}}$ is the first PiolaKirchhoff stress tensor evaluated at $\mathscr{S}$. This hypothesis is sustained on the physical perception

11 that if there are material points in between $\Omega^{+}$and $\Omega^{-}$the traction continuity (equilibrium) should be also extended to those points.

13 The nominal traction continuity condition (14) leads to the requirement of a bounded character for the Cauchy stress tensor at the interior of the discontinuity interface, $\boldsymbol{\sigma}_{\mathscr{S}}$,

15 and also for its time derivative $\dot{\boldsymbol{\sigma}}_{\mathscr{g}}$. This requirements emerge from the following reasonings:

17 1. Since the deformation at $\Omega \backslash \mathscr{S}$ is determined by $\overline{\mathbf{F}}$ (that is bounded by definition, according to Equation (3)), and the continuum constitutive equation is supposed to re-

19 turn bounded stresses for bounded strains, then the Piola-Kirchhoff stresses $\mathbf{P}_{\Omega^{+}}(\overline{\mathbf{F}})$ and $\mathbf{P}_{\Omega^{-}}(\overline{\mathbf{F}})$ must be bounded at any time of the analysis.

21 2. If $\mathbf{P}_{\Omega^{+}}$and $\mathbf{P}_{\Omega^{-}}$are bounded, so must be the nominal traction vector $\mathscr{T}$ in Equation (14) since $\mathbf{N}$ is bounded $(|\mathbf{N}|=1)$.

23 3. Rewriting the last Equation (14) in terms of the Cauchy stresses $\boldsymbol{\sigma}_{\mathscr{S}}$ one gets:

$$
\mathscr{T}=\mathbf{P}_{\mathscr{S}} \cdot \mathbf{N}=J \boldsymbol{\sigma}_{\mathscr{S}} \cdot \mathbf{n}=\bar{J}_{\mathscr{\sigma}} \cdot \overline{\mathbf{n}}
$$

25 where Equations (10) and (12) have been used. Hence, since, $\bar{J}$ and $\overline{\mathbf{n}}$ are bounded entities, if all the components of $\boldsymbol{\sigma}_{\mathscr{S}}$ were bounded so would be their linear combinations $\mathscr{T}_{i}=\bar{J} \boldsymbol{\sigma}_{i j} \bar{n}_{j}$ defining Equation (15).

4. Similar arguments, now on rate entities, lead to require the bounded character of $\dot{\boldsymbol{\sigma}}_{\mathscr{S}}$. In fact, if $\dot{\mathbf{P}}_{\Omega^{+}}$and $\dot{\mathbf{P}}_{\Omega^{-}}$are assumed to be bounded on the same above arguments, time derivation of Equation (15), considering the material character of $\mathscr{S}(\mathbf{N}=0)$, leads to 


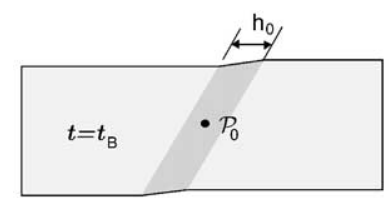

(a)

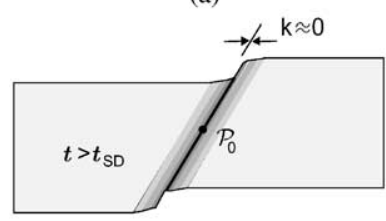

(c)

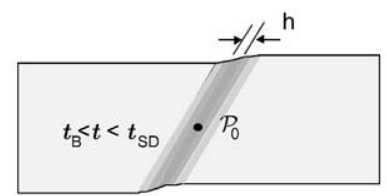

(b)

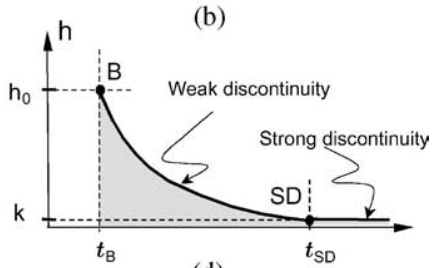

(d)

Figure 3. From (a) to (c): mechanism of formation of a strong discontinuity by collapse of a weak discontinuity; and (d) variable bandwidth law.

the bounded character of $\dot{\mathscr{T}}$ :

$$
\underbrace{\dot{\mathscr{T}}}_{\text {bounded }}=\dot{\bar{J}}_{\boldsymbol{\sigma}_{\mathscr{S}}} \cdot \overline{\mathbf{n}}+\bar{J} \dot{\boldsymbol{\sigma}}_{\mathscr{S}} \cdot \mathbf{n}+\bar{J}_{\boldsymbol{\sigma}} \cdot \dot{\overline{\mathbf{n}}}
$$

Since $\dot{\bar{J}}$ and $\dot{\overline{\mathbf{n}}}$ (from time derivation of the last Equation (5)) are bounded entities, the bounded character of $\dot{\mathscr{T}}$ is guaranteed if $\dot{\boldsymbol{\sigma}}_{\mathscr{S}}$ is also bounded.

5 Therefore, boundedness of $\boldsymbol{\sigma}_{\mathscr{S}}$ and $\dot{\boldsymbol{\sigma}}_{\mathscr{S}}$ guarantees the boundedness of $\mathscr{T}$ and $\dot{\mathscr{T}}$ that is demanded from a physical viewpoint. The main goal of the Strong Discontinuity Analysis,

7 developed in Section 3.5.1, is precisely to determine the ingredients that have to be introduced in a continuum constitutive model to guarantee that bounded character even in presence of

9 unbounded strain measures.

\subsection{Development of a strong discontinuity. Weak-strong discontinuities}

11 The regularized kinematics proposed in Section 2.1, allows to introduce the weak discontinuity concept by considering the same kinematics in Equations (7)-(12) but now with a non-

13 null bandwidth** $h \neq 0$. Bearing these concepts in mind, we shall consider the mechanism of formation of a strong discontinuity as follows:

15 (a) at time $t=t_{\mathrm{B}}$ (the bifurcation time) a local discontinuous bifurcation of the strain field (see Section 3.3) triggers a localization of the strains in the shape of a weak discontinuity (with 17 bandwidth $h=h_{0}$ ), see Figure 3(a).

(b) a subsequent evolution of the bandwidth $h(t)$, decreasing monotonously along time, makes that weak discontinuity collapse into a strong discontinuity (when the bandwidth reaches a very small regularization value $h \equiv k \rightarrow 0$ ) at time $t_{\mathrm{SD}}$ (see Figure $3(\mathrm{~b})-(\mathrm{c})$ ). For the

\footnotetext{
**A weak discontinuity can be then characterized by continuous displacements fields and discontinuous (but bounded) strain fields [25].
} 


\section{NME 607}

1 strong discontinuity regime $\left(t \geqslant t_{\mathrm{SD}}\right)$ the bandwidth is kept constant, $h \equiv k \rightarrow 0$, (see Figure $3(\mathrm{~d}))$.

3 The variable bandwidth law of Figure 3(d) is a model ingredient, whose fundamental role in the continuum approach (SDA) has already been explored in infinitesimal strain settings

5 [18]. It is a mechanism for delaying the onset of a strong discontinuity after the bifurcation time $t_{\mathrm{B}}$ till the stress state satisfies the, necessary, strong discontinuity conditions derived in

7 Section 3.5.1. From the mechanical point of view, the transition (weak discontinuity) regime $\left[t_{\mathrm{B}}, t_{\mathrm{SD}}\right]$ defines in the tip of a propagating discontinuity a zone where the discontinuity is

9 processed that can be readily identified with the Fracture Process Zone concept in Fracture Mechanics [26]. The bandwidth evolution is considered a material property defined in terms

11 of the stress-like internal variable of the continuum constitutive equation. More details can be found in References [18, 25].

\section{3.3. Bifurcation condition at $t=t_{\mathrm{B}}$}

By resorting to the so-called discontinuous bifurcation analysis $[27,28]$ we can determine the

15 conditions for the bifurcation of an initially smooth deformation field into a weak discontinuity compatible, in turn, with the equilibrium of the body. Therefore, we assume that at time $t=t_{\mathrm{B}}$

17 a non-smooth deformation rate, described by the rate of the deformation gradient (7), begins developing. The equilibrium condition (14) across the discontinuity surface $\mathscr{S}$ requires the

19 jump of the rate of the nominal traction vector to be zero:

$$
\llbracket \dot{\mathscr{T}} \rrbracket=\left[\dot{\mathbf{P}}\left(\mathbf{X}_{\mathscr{S}}\right)-\dot{\mathbf{P}}\left(\mathbf{X}_{\Omega^{+}}\right)\right] \cdot \mathbf{N}=\mathbf{0}
$$

21 Assuming loading conditions in $\mathscr{S}$ and neutral loading in $\Omega \backslash \mathscr{S}$, $^{\dagger \dagger}$ and after some algebraic manipulations, it is possible to derive from (17) the following equation [29]:

$$
\mathbf{Q}^{L} \cdot \llbracket \dot{\mathbf{u}} \rrbracket=\underbrace{\left(\mathbf{e}_{n} \cdot \mathbf{c}^{\mathrm{tang}} \cdot \mathbf{e}_{n}+\left(\mathbf{e}_{n} \cdot \tau \cdot \mathbf{e}_{n}\right) \mathbb{1}\right)}_{\mathbf{Q}^{L}} \cdot \llbracket \dot{\mathbf{u}} \rrbracket=\mathbf{0}
$$

where $^{\text {tang }}$ is the tangent constitutive tensor, which relates the Kirchhoff stress convective rate

25 with the rate of deformation $\left(L_{v} \tau=\mathbf{c}^{\text {tang }}: \mathbf{d}\right.$, see the appendix for applications to a particular model). The criterion to determine bifurcation is based on the detection of the singularity of

27 the localization tensor $\mathbf{Q}^{L}$, this allowing a non-trivial solution for the velocity jump $(\llbracket \dot{\mathbf{u}} \rrbracket \neq 0)$ in Equation (18):

$$
\operatorname{det}\left(\mathbf{Q}^{L}\left(\mathbf{e}_{n}, \mathscr{H}^{\text {crit }}\right)\right)=0 \quad \text { for } t=t_{\mathrm{B}}
$$

where $\mathscr{H}^{\text {crit }}$ is the maximum (critical) value of the softening modulus compatible with Equa31 tion (19). The first time that this equation is fulfilled for a given material point,

\footnotetext{
${ }^{\dagger}$ A preliminary analysis shows that this scenario determines the first (and, therefore, the most unfavourable) possible bifurcation.
} 
1 determines the bifurcation time $t_{\mathrm{B}}$ for that point, and allows to obtain the normal $\mathbf{e}_{n}$, which in turn determines the direction of propagation of the discontinuity interface $\mathscr{S}$. For further

3 details on this particular procedure the reader is referred, for instance, to References [28-30].

\subsection{The continuum and discrete free energies}

5 There is a broad set of continuum constitutive models founded on thermodynamic basis that can be used in finite strain settings. A key point in those models is the definition of the

7 continuum free energy density function $\psi(\mathbf{F}, \Gamma)$, in terms of the gradient of deformation tensor $\mathbf{F}$, that acts as the free (thermodynamically independent) variable, and a set of internal

9 variables $\boldsymbol{\Gamma}$ (including the inelastic strain measures) characterized by specific evolution laws [31]. The nominal stress field $\mathbf{P}$ can be then directly obtained, on thermodynamical reasonings,

11 from that continuum free energy as

$$
\mathbf{P}(\mathbf{X}, t)=\frac{\partial \psi(\mathbf{F}, \boldsymbol{\Gamma})}{\partial \mathbf{F}}:=\partial \psi_{\mathbf{F}}(\mathbf{F}, \boldsymbol{\Gamma})
$$

13 which qualifies the continuum free energy $\psi$ as a potential for the nominal stress field $\mathbf{P}$.

In this context, let us consider the discontinuous interface $\mathscr{S}$ and the free energy per unit

15 of this surface, $\bar{\psi}$, from now on termed discrete free energy which, in the context of the regularization procedure sketched in Figure 1(b), can be written as

$$
\bar{\psi}=\frac{\text { free energy }}{\text { unit surface }}=\underbrace{\frac{\text { free energy }}{\text { unit volume }}}_{\psi} \cdot \underbrace{\frac{\text { unit volume }}{\text { unit surface }}}_{h}=\left.\lim _{h \equiv k \rightarrow 0} h \psi\right|_{\mathscr{S}}
$$

Now, by considering the strong discontinuity kinematics $(8), \mathbf{F}(\overline{\mathbf{F}}, \boldsymbol{\beta})=\overline{\mathbf{F}}+\mu_{\mathscr{S}} / h(\boldsymbol{\beta} \otimes \mathbf{N})$, in 19 Equation (20) one gets:

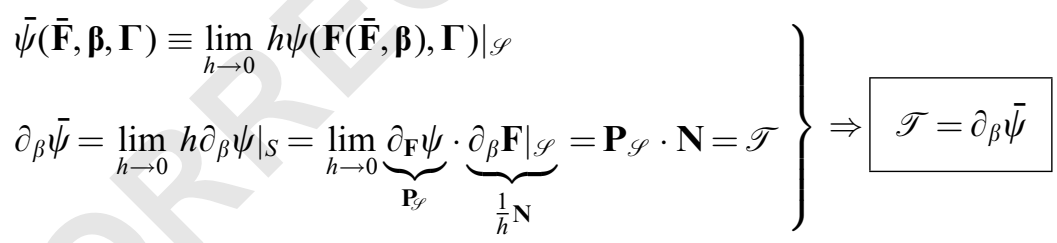

21 Equation (22) hints at a crucial consequence of the insertion of strong discontinuous kinematics into continuum (stress-strain) models: the projection of those continuum models into

23 discrete (traction-displacement jump) ones. In fact, the discrete free energy $\bar{\psi}$, obtained as the discontinuous surface counterpart of the continuum free energy density $\psi$, turns into a

25 potential of the nominal traction $\mathscr{T}=\mathbf{P}_{\mathscr{S}} \cdot \mathbf{N}$, with respect to the incremental jump $\boldsymbol{\beta}$, as shown in Equation (22). This suggests that a discrete model can be derived from that discrete free

27 energy and, therefore, from the inclusion of a strong discontinuity kinematics in the original continuum model. Indeed, this is what is shown, for a target constitutive model (continuum damage), in next sections. 


\section{NME 607}

13.5 . A representative continuum damage model

3 Let us now consider the extension to the finite deformation range of the isotropic continuum damage model presented in [17]:

\begin{tabular}{|c|c|}
\hline Free energy & $\begin{array}{c}\psi(\mathbf{b}, r)=(1-d) \psi^{0}(\mathbf{b}) \\
\psi^{0}(\mathbf{b})=\frac{1}{4} \lambda\left(J^{2}-1\right)-\left(\frac{\lambda}{2}+\mu\right) \log J+\frac{1}{2} \mu[\operatorname{tr}(\mathbf{b})-3]\end{array}$ \\
\hline $\begin{array}{l}\text { Constitutive } \\
\text { equation }\end{array}$ & $\mathbf{P}=\partial_{\mathbf{F}} \psi \Leftrightarrow \tau=2 \mathbf{b} \cdot \partial_{\mathbf{b}} \psi=\frac{q}{r}\left[\lambda \frac{\left(J^{2}-1\right)}{2} \mathbb{1}+\mu(\mathbf{b}-\mathbb{1})\right]$ \\
\hline $\begin{array}{l}\text { Damage } \\
\text { variable }\end{array}$ & $d=1-\frac{q(r)}{r} ; d \in[0,1]$ \\
\hline $\begin{array}{l}\text { Evolution } \\
\text { law }\end{array}$ & $\dot{r}=\gamma\left\{\begin{array}{l}r \in\left[r_{0}, \infty\right) \\
\left.r\right|_{t=0}=r_{0}>0\end{array}\right.$ \\
\hline $\begin{array}{l}\text { Damage } \\
\text { criterion }\end{array}$ & $\phi(\boldsymbol{\sigma}, q) \equiv \tau_{\sigma}-q ; \tau_{\boldsymbol{\sigma}}=\sqrt{\boldsymbol{\sigma}: \mathbf{c}_{\phi}^{-1}: \boldsymbol{\sigma}}$ \\
\hline $\begin{array}{l}\text { Load.-unl. } \\
\text { conditions }\end{array}$ & $\gamma \geqslant 0 \quad \phi \leqslant 0 \quad \gamma \phi=0$ \\
\hline $\begin{array}{l}\text { Softening } \\
\text { rule }\end{array}$ & $\dot{q}=\mathscr{H} \dot{r} \quad \mathscr{H}<0 \quad\left\{\begin{array}{l}q \in\left[0, q_{0}\right] \\
q_{0}:=\left.q\right|_{t=0}=r_{0}\end{array}\right.$ \\
\hline
\end{tabular}

where $\lambda$ and $\mu$ are the Lame's parameters, $\tau$ is the Kirchhoff stress tensor, $\mathbf{b}(\mathbf{F})=\mathbf{F} \cdot \mathbf{F}^{\mathrm{T}}$ is

5 the left Cauchy-Green deformation tensor, $r$ is a scalar strain-like internal variable which determines the damage (or degradation) level of the material and $q(r)$ is a stress-like internal

7 variable (hardening variable) that sets the evolution of the elastic domain $E_{\sigma}:=\{\boldsymbol{\sigma} ; \phi(\boldsymbol{\sigma}, q)<0\}$ through the damage function $\phi(\sigma, q)$. The initial value of $r$ is $r_{0}=\sigma_{u} / \sqrt{E}$ where $\sigma_{u}$ is the

9 uniaxial peak stress and $E$ is Young modulus. In addition, in Equation $(23 \mathrm{c}), d(r)=1-q(r) / r$ is the classical damage variable ranging from 0 (undamaged state) to 1 (full damage). Also

11 in Equation (23) $\tau_{\sigma}$ is a norm of the stresses in the metric of the tensor $\mathbf{c}_{\phi}^{-1}, \gamma$ is the damage multiplier and $\mathscr{H}$ is the softening modulus from now on termed continuum softening modulus

13 (see the appendix for the explicit expression of tensor $\mathbf{c}_{\phi}^{-1}$ and additional details on the model).

3.5.1. Strong discontinuity analysis. Let us find out what conditions make the unbounded

15 strains at the strong discontinuity regime, $t \geqslant t_{\mathrm{SD}}$ (and, thus, $h \equiv k \rightarrow 0$ ), compatible with the stress boundedness requirements of Section 3.1. Using the multiplicative decomposition (5)

17 and expressions (8) and (10), one can rewrite the Kirchhoff stress (23b) in the discontinuous interface $\mathscr{S}$ as

19

$$
\tau_{\mathscr{S}}=\frac{q_{\mathscr{S}}}{r_{\mathscr{S}}}\left[\frac{\lambda}{2}\left(\bar{J}^{2}\left[\frac{k+\boldsymbol{\beta} \cdot \overline{\mathbf{n}}}{k}\right]^{2}-1\right) \mathbb{1}+\mu(\overline{\mathbf{b}}-\mathbb{1})+2 \mu\left(\frac{\overline{\mathbf{b}} \cdot \overline{\mathbf{n}} \otimes \boldsymbol{\beta}}{k}\right)^{\mathrm{sym}}+\mu\left(\frac{\boldsymbol{\beta} \otimes \boldsymbol{\beta}}{k^{2}}\right)\right]
$$




\section{NME 607}

1 where $\overline{\mathbf{b}}=\overline{\mathbf{F}} \cdot \overline{\mathbf{F}}^{\mathrm{T}}$. The corresponding Cauchy stresses, taking into account Equations (10) and (11), and after some algebraic manipulations can be written as

$$
\boldsymbol{\sigma}_{\mathscr{S}}=\frac{1}{J_{\mathscr{S}}} \tau_{\mathscr{S}}=\frac{q_{\mathscr{S}}}{r_{\mathscr{S}}}\left(\boldsymbol{\sigma}_{0}+\frac{1}{k} \boldsymbol{\sigma}_{1}\right)=\frac{q_{\mathscr{S}}}{k r_{\mathscr{S}}}\left(k \boldsymbol{\sigma}_{0}+\boldsymbol{\sigma}_{1}\right)
$$

where

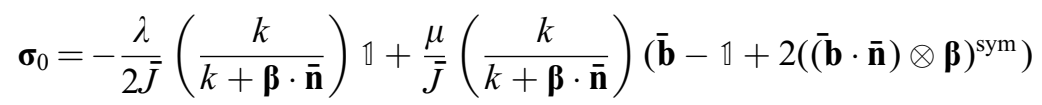

and

$$
\boldsymbol{\sigma}_{1}=\frac{\lambda}{2} \bar{J}(k+\boldsymbol{\beta} \cdot \overline{\mathbf{n}}) \mathbb{1}+\frac{\mu}{\bar{J}}\left(\frac{\boldsymbol{\beta} \otimes \boldsymbol{\beta}}{k+\boldsymbol{\beta} \cdot \overline{\mathbf{n}}}\right)
$$

From Equation (25), and for the strong discontinuity regime $\left(t \geqslant t_{\mathrm{SD}} h \equiv k \rightarrow 0\right)$, one gets:

$$
\boldsymbol{\sigma}_{\mathscr{S}}=\frac{q_{\mathscr{S}}}{\lim _{k \rightarrow 0} k r_{\mathscr{S}}} \lim _{k \rightarrow 0}(\underbrace{k \boldsymbol{\sigma}_{0}}_{=0}+\boldsymbol{\sigma}_{1})=\frac{q_{\mathscr{S}}}{\lim _{k \rightarrow 0} k r_{\mathscr{S}}}\left[\frac{\lambda}{2} \bar{J}(\boldsymbol{\beta} \cdot \overline{\mathbf{n}}) \mathbb{1}+\frac{\mu}{\bar{J}(\boldsymbol{\beta} \cdot \overline{\mathbf{n}})} \boldsymbol{\beta} \otimes \boldsymbol{\beta}\right]
$$

where the bounded character of $\boldsymbol{\sigma}_{0}$ (from inspection of Equation (26)), and then $\lim _{k \rightarrow 0}\left(k \boldsymbol{\sigma}_{0}\right)$

$11=\mathbf{0}$ has been considered. Multiplying Equation (28) times $\bar{J} \overline{\mathbf{n}}$ and recalling Equation (25) $\left(\mathscr{T}=\bar{J} \boldsymbol{\sigma}_{\mathscr{S}} \cdot \overline{\mathbf{n}}\right)$ we obtain:

$$
\mathscr{T}=\bar{J} \boldsymbol{\sigma}_{\mathscr{S}} \cdot \overline{\mathbf{n}}=\frac{1}{\lim _{k \rightarrow 0}\left(k r_{\mathscr{S}}\right)} q_{\mathscr{S}}\left[\frac{\lambda}{2} \bar{J}^{2}(\boldsymbol{\beta} \cdot \overline{\mathbf{n}}) \overline{\mathbf{n}}+\mu \boldsymbol{\beta}\right]
$$

In view of Equation (29) we now consider the following two scenarios:

(I) $\lim _{k \rightarrow 0}\left(k r_{\mathscr{S}}\right)=0$ for $t>t_{\mathrm{SD}}\left(r_{\mathscr{S}}=\right.$ bounded $)$. Then, from Equation (29) the bounded character of $\mathscr{T}$ implies:

$$
\frac{\lambda}{2} \bar{J}^{2}(\boldsymbol{\beta} \cdot \overline{\mathbf{n}}) \overline{\mathbf{n}}+\mu \boldsymbol{\beta}=\mathbf{0} \quad \text { for } t \geqslant t_{\mathrm{SD}}
$$

and multiplying Equation (30) times $\overline{\mathbf{n}}$ :

$$
\underbrace{\left(\frac{\lambda}{2} \bar{J}^{2}|\overline{\mathbf{n}}|^{2}+\mu\right)}_{\neq 0}(\boldsymbol{\beta} \cdot \overline{\mathbf{n}})=0 \Leftrightarrow(\boldsymbol{\beta} \cdot \overline{\mathbf{n}})=0 \Leftrightarrow \boldsymbol{\beta}=\mathbf{0} \quad \text { for } t \geqslant t_{\mathrm{SD}}
$$

where Equation (30) and the facts $\lambda \geqslant 0, \mu>0$ have been considered. Equation (31) states that the incremental displacement jump $\boldsymbol{\beta}$ is null and, thereby, no evolution of the jump at the strong discontinuity regime. Therefore, this scenario would not model the strong discontinuity and has to be discarded. The alternative scenario is then: 


\section{NME 607}

1 (II) $\lim _{k \rightarrow 0}\left(k r_{\mathscr{S}}\right) \neq 0$ for $\forall t>t_{\mathrm{SD}}\left(r_{\mathscr{S}}=\mathcal{O}(1 / k)=\right.$ unbounded $)$ : Such condition is trivially fulfilled for the following structure of $\dot{r}_{\mathscr{S}}$, in Equation (23d):

$$
\begin{aligned}
& \dot{r}_{\mathscr{S}}=\gamma=\frac{\dot{\bar{\alpha}}}{k} \geqslant 0 \quad \forall t \geqslant t_{\mathrm{SD}}
\end{aligned}
$$

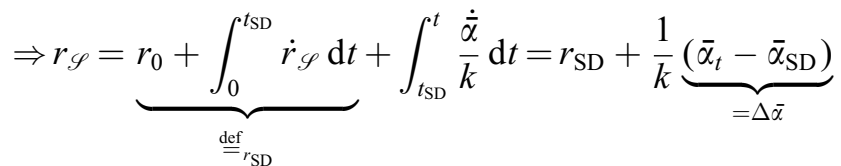

5 where $\dot{\bar{\alpha}}$ and, therefore, $\bar{\alpha}$ and $\Delta \bar{\alpha}$ are bounded and Equation (23f) $(\gamma \geqslant 0)$ has been considered. From now on the variable $\bar{\alpha}$ will be termed discrete internal variable. From Equations (32) and $(33)$ :

$$
\left.\begin{array}{c}
\dot{\bar{\alpha}}=k \underbrace{\gamma}_{\geqslant 0} \stackrel{\text { def }}{=} \bar{\gamma} \geqslant 0 \\
\Delta \bar{\alpha} \stackrel{\text { def }}{=} \bar{\alpha}_{t}-\bar{\alpha}_{\mathrm{SD}} \geqslant 0
\end{array}\right\} \forall t>t_{\mathrm{SD}}
$$

9 where the parameter $\bar{\gamma}$ will be termed discrete damage multiplier.

From Equation (33) it follows that the assumption for scenario (II) is fulfilled, that is

$$
\lim _{k \rightarrow 0}\left(k r_{\mathscr{S}}\right)=\underbrace{\lim _{k \rightarrow 0} k r_{\mathrm{SD}}}_{=0}+\left(\bar{\alpha}_{t}-\bar{\alpha}_{\mathrm{SD}}\right)=\Delta \bar{\alpha}>0 \quad \forall t>t_{\mathrm{SD}}
$$

Let us now consider the hardening/softening variable $q_{\mathscr{S}}$ that, from Equation $(23 \mathrm{~g})\left(q \in\left[0, r_{0}\right]\right)$,

13 is bounded. Let us find out what conditions would make also $\dot{q}_{\mathscr{S}}$ bounded. From the softening rule $(23 \mathrm{~g})$, in connection with Equation (32) for $t \geqslant t_{\mathrm{SD}}$, and loading cases we obtain:

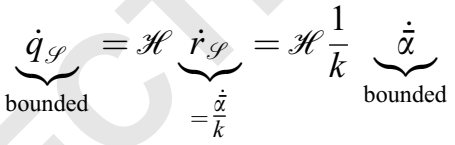

Hence, the continuum softening modulus $\mathscr{H}$ must fulfill:

$$
\mathscr{H} \frac{1}{k}=\overline{\mathscr{H}} \text { (bounded) }
$$

and substitution of Equation (37) into Equation (36) leads to

$$
\dot{q}_{\mathscr{S}}=\overline{\mathscr{H}} \dot{\bar{\alpha}} \quad \forall t \geqslant t_{\mathrm{SD}}
$$

which constitutes a discrete softening rule in terms of the discrete internal variable $\bar{\alpha}$. On the other hand Equation (37) is fulfilled from the following softening regularization condition: ${ }^{\ddagger}$

$$
\mathscr{H}=h \overline{\mathscr{H}} \quad \forall t \geqslant t_{\mathrm{B}}
$$

\footnotetext{
$¥ \ddagger$ In strict sense the softening regularization condition is only required at the strong discontinuity regime ( $\mathscr{H}=k \overline{\mathscr{H}} \quad \forall t \geqslant t_{\mathrm{SD}}$ ) but, in the context of the variable bandwidth model, it is also extended to the weak discontinuity regime $\left(t_{\mathrm{B}} \leqslant t<t_{\mathrm{SD}}\right)$ (see References [18, 25]).
} 
1 where $\overline{\mathscr{H}}<0$ (from now on termed the discrete softening modulus) is a (bounded) material parameter. $^{\S \S}$

3 Finally, substitution of Equation (35) into (28) leads to

$$
\boldsymbol{\sigma}_{\mathscr{S}}\left(q_{\mathscr{S}}, \Delta \bar{\alpha}, \boldsymbol{\beta}\right)=\frac{q_{\mathscr{S}}}{\Delta \bar{\alpha}}\left[\frac{\lambda}{2} \bar{J}(\boldsymbol{\beta} \cdot \overline{\mathbf{n}}) \mathbb{1}+\mu \frac{1}{\bar{J} \boldsymbol{\beta} \cdot \overline{\mathbf{n}}} \boldsymbol{\beta} \otimes \boldsymbol{\beta}\right] \quad \forall t \geqslant t_{\mathrm{SD}}
$$

5 Equation (40) guaranties the bounded character of $\boldsymbol{\sigma}_{\mathscr{S}}$ as a continuous function of the bounded entities $q_{\mathscr{S}}, \Delta \bar{\alpha}$ and $\boldsymbol{\beta}$. Moreover, time derivation of Equation (40) also shows the bounded

7 character of $\dot{\boldsymbol{\sigma}}_{\mathscr{C}}\left(q \mathscr{S}, \Delta \bar{\alpha}, \boldsymbol{\beta}, \dot{q}_{\mathscr{S}}, \dot{\bar{\alpha}}, \dot{\boldsymbol{\beta}}\right)$ since $\dot{q}_{\mathscr{S}}, \dot{\bar{\alpha}}$ and $\dot{\boldsymbol{\beta}}$ are also bounded. Therefore, it appears that the softening regularization in Equation (39) is a sufficient condition to guarantee the bounded character of the stress and rate of stress fields required in Section 3.1.

On the other hand, Equation (40) provides a set of six (due to its symmetry) equations that

11 allows to solve for the incremental displacement jump $\boldsymbol{\beta}$ (three equations) and also supplies three additional constrains on the stress field $\boldsymbol{\sigma}_{\mathscr{S}}$. Indeed, multiplying such equation times $\bar{J} \overline{\mathbf{n}}$,

13 and considering Equation (15) $\left(\mathscr{T}=\bar{J}_{\mathscr{S}} \cdot \overline{\mathbf{n}}\right)$ one obtains:

$$
\mathscr{T}=\bar{J} \boldsymbol{\sigma}_{\mathscr{S}} \cdot \overline{\mathbf{n}}=\frac{q_{\mathscr{S}}}{\Delta \bar{\alpha}} \underbrace{\left(\frac{\lambda}{2} \bar{J}^{2}(\overline{\mathbf{n}} \otimes \overline{\mathbf{n}})+\mu \mathbb{1}\right)}_{\mathbf{Q}} \cdot \boldsymbol{\beta}=\frac{q_{\mathscr{S}}}{\Delta \bar{\alpha}} \mathbf{Q} \cdot \boldsymbol{\beta}
$$

15 or, equivalently:

$$
\mathscr{T}=(1-\omega) \mathbf{Q} \cdot \boldsymbol{\beta} ; \quad \omega=1-\frac{q_{S}}{\Delta \bar{\alpha}} ; \quad \omega \in[-\infty, 1] \quad \forall t \geqslant t_{\mathrm{SD}}
$$

17 that can clearly be interpreted as a discrete damage constitutive equation for the cohesive discontinuous interface $\mathscr{S}$. It describes the relation between the traction $\mathscr{T}$ and the displacement

19 jump $\boldsymbol{\beta}$ in terms of a discrete damage variable $\omega \in[-\infty, 1]^{\top \uparrow}$ and an acoustic-like stiffness tensor $\mathbf{Q}$.

Equation (42) can be solved for $\boldsymbol{\beta}$ (as $\left.\boldsymbol{\beta}=\left(\Delta \bar{\alpha} / q_{S}\right) \mathbf{Q}^{-1} \cdot \mathscr{T}\right)$ and, once substituted into Equation (40), provides, after some algebraic manipulations, a set of three equations in terms of

$23 \boldsymbol{\sigma}_{\mathscr{S}}$. These conditions on the stress field, which are termed the strong discontinuity conditions [17], have to be fulfilled at the strong discontinuity regime ${ }^{\|\|}\left(t \geqslant t_{\mathrm{SD}}\right)$. In a local orthogonal

25 basis $\left(\mathbf{e}_{1}, \mathbf{e}_{2}, \mathbf{e}_{3}\right)$, see Figure 4 , with unit vectors $\mathbf{e}_{2}$ and $\mathbf{e}_{3}$ laying on the tangent plane to the

\footnotetext{
$\S \S$ In fact the discrete softening parameter $\overline{\mathscr{H}}$ can be readily related to the fracture energy concept in fracture mechanics [17].

T The initial $\omega=-\infty$ value states that the induced discrete model is a rigid damage model. This extends to finite deformation settings this feature already proved for infinitesimal strains settings [17].

"II As it will be shown through numerical simulations in Section 5.1, the strong discontinuity conditions (43) are not always fulfilled at the bifurcation time $t_{\mathrm{B}}$ and, in the context of the SDA, this fact generally precludes the onset of the strong discontinuity immediately after the bifurcation, see References $[17,25]$ for additional details. The variable bandwidth (weak-strong discontinuity) model outlined in Section 3.2 constitutes a mechanism to smoothly induce those conditions that, in turn, can be related to the Fracture Process Zone concept in Fracture Mechanics $[25,26]$.
} 


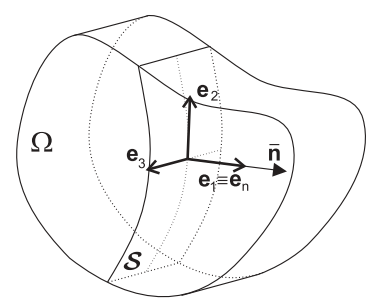

Figure 4. Orthogonal basis attached to the discontinuity surface.

1 discontinuity surface, $\mathscr{S}$, and $\mathbf{e}_{1}=\mathbf{n} /\|\mathbf{n}\|=\overline{\mathbf{n}} /\|\overline{\mathbf{n}}\|$, they can be written as:

$$
\left.\begin{array}{l}
\sigma_{22_{s}}=\frac{\hat{\lambda}}{\mu+\hat{\lambda}} \sigma_{11_{s}}+\frac{\mu+\hat{\lambda}}{\mu}\left(\sigma_{12_{s}}^{2} / \sigma_{11_{s}}\right) \\
\sigma_{33_{s}}=\frac{\hat{\lambda}}{\mu+\hat{\lambda}} \sigma_{11_{s}}+\frac{\mu+\hat{\lambda}}{\mu}\left(\sigma_{13_{s}}^{2} / \sigma_{11_{s}}\right) \quad\left(\hat{\lambda}=\frac{\lambda}{2} \bar{J}^{2}\|\overline{\mathbf{n}}\|^{2}\right) \\
\sigma_{23_{s}}=\frac{\mu+\hat{\lambda}}{\mu}\left(\sigma_{12_{s}} \sigma_{13_{s}} / \sigma_{11_{s}}\right)
\end{array}\right\} \forall t \geqslant t_{\mathrm{SD}}
$$

3 which states a continuous functional dependence of the stress $\sigma_{22_{S}}, \sigma_{33_{S}}$ and $\sigma_{23_{S}}$ on the traction vector components $[\mathscr{T}] \equiv\left[\sigma_{11_{S}}, \sigma_{12_{S}}, \sigma_{13_{S}}\right]$. Hence, all the components of $\boldsymbol{\sigma}_{\mathscr{S}}$ can be expressed

5 as a function of the components of $\mathscr{T}$, i.e.

$$
\boldsymbol{\sigma}_{\mathscr{S}}=\boldsymbol{\sigma}(\mathscr{T}) \quad \forall t \geqslant t_{\mathrm{SD}}
$$

7 Considering now the $\mathscr{T}$-dependence of $\boldsymbol{\sigma}_{\mathscr{S}}$ stated in Equation (44), and substituting into the damage criterion $(23 \mathrm{e})$ we obtain:

$$
\bar{\phi}\left(\mathscr{T}, q_{\mathscr{S}}\right) \stackrel{\text { def }}{=} \phi\left(\boldsymbol{\sigma}(\mathscr{T}), q_{\mathscr{S}}\right) \equiv \tau_{\mathscr{T}}-q_{\mathscr{S}} ; \quad \tau_{\mathscr{T}}=\sqrt{\boldsymbol{\sigma}(\mathscr{T}): \mathbf{c}_{\phi}^{-1}: \boldsymbol{\sigma}(\mathscr{T})} \quad \forall t \geqslant t_{\mathrm{SD}}
$$

which constitutes a discrete damage criterion at the interface in terms of the traction $\mathscr{T}$.

11 Finally, recalling the expression of the discrete free energy (21) for the particular case of Equations (23a) one obtains, after some algebraic manipulation:

$$
\begin{aligned}
\lim _{h \equiv k \rightarrow 0} k \psi(\mathbf{b}, r) & =\bar{\psi}(\boldsymbol{\beta}, \Delta \bar{\alpha}) \\
\bar{\psi}(\boldsymbol{\beta}, \Delta \bar{\alpha}) & =\frac{q_{\mathscr{S}}(\Delta \bar{\alpha})}{\Delta \bar{\alpha}} \bar{\psi}^{0}(\boldsymbol{\beta}) ; \quad \bar{\psi}^{0}=\frac{\lambda}{4} \bar{J}^{2}(\boldsymbol{\beta} \cdot \overline{\mathbf{n}})^{2}+\frac{\mu}{2}(\boldsymbol{\beta} \cdot \boldsymbol{\beta})
\end{aligned}
$$

13 and, from the expression of the discrete free energy (47), the constitutive Equation (41) is immediately recovered by derivation $\left(\mathscr{T}=\partial_{\beta} \bar{\psi}\right)$, as suggested in Section 3.4. In summary, by

15 collecting the expressions derived in this section, the following discrete constitutive model at the discontinuous interface $\mathscr{S}$ emerges (Figure 5): 

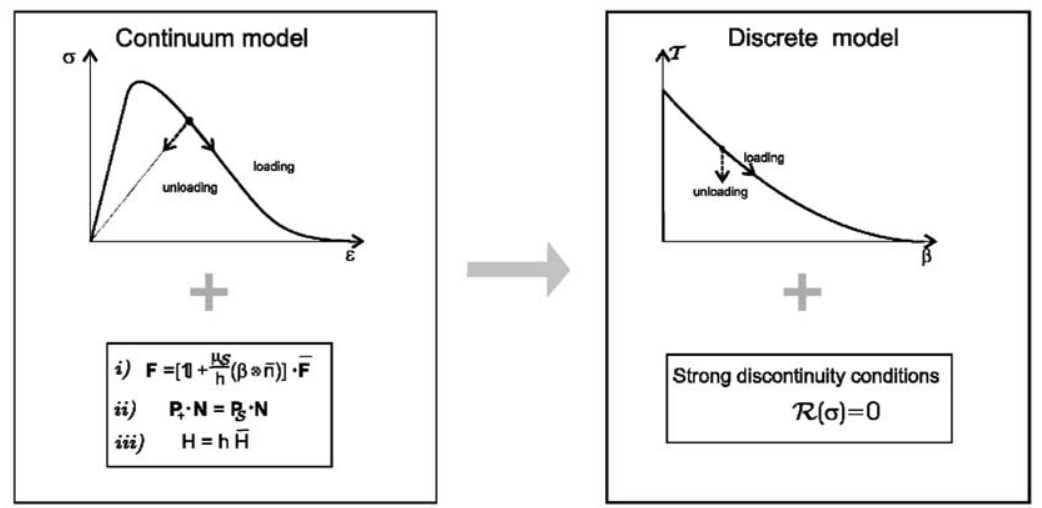

(a)

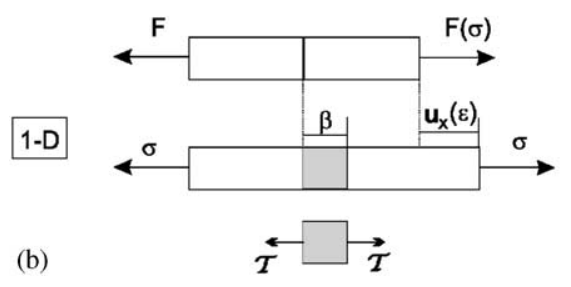

2-D

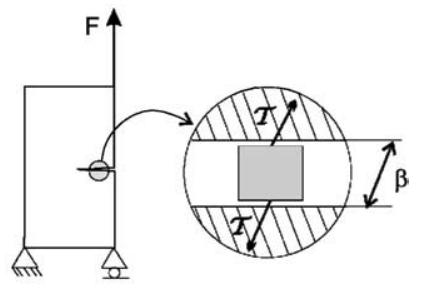

(c)

Figure 5. (a) Original (continuum) vs induced (discrete) constitutive response; (b) and (c) induced discontinuous interfaces.

\begin{tabular}{|c|c|}
\hline Free energy & $\bar{\psi}(\beta, \Delta \bar{\alpha})=(1-\omega) \bar{\psi}^{0} ; \quad\left\{\begin{array}{l}\bar{\psi}^{0}(\boldsymbol{\beta})=\frac{1}{2} \boldsymbol{\beta} \cdot \mathbf{Q} \cdot \boldsymbol{\beta} \\
\mathbf{Q}=\frac{\lambda}{2} \bar{J}^{2}(\overline{\mathbf{n}} \otimes \overline{\mathbf{n}})+\mu \mathbb{1}\end{array}\right.$ \\
\hline $\begin{array}{l}\text { Constitutive } \\
\text { equation }\end{array}$ & $\mathscr{T}=\partial_{\beta} \bar{\psi}=(1-\omega) \mathbf{Q} \cdot \boldsymbol{\beta}$ \\
\hline $\begin{array}{l}\text { Damage } \\
\text { variable }\end{array}$ & $\omega=1-\frac{q_{\mathscr{S}}(\Delta \bar{\alpha})}{\Delta \bar{\alpha}} ; \quad \omega \in(-\infty, 1]$ \\
\hline $\begin{array}{l}\text { Evolution } \\
\text { law }\end{array}$ & $\dot{\bar{\alpha}}=\bar{\gamma} ; \quad\left\{\begin{array}{l}\Delta \bar{\alpha} \in[0, \infty) \\
\left.\Delta \bar{\alpha}\right|_{t_{\mathrm{SD}}}=0\end{array}\right.$ \\
\hline $\begin{array}{l}\text { Damage } \\
\text { criterion }\end{array}$ & $\bar{\phi}(\mathscr{T}, q)=\tau_{\mathscr{T}}-q_{\mathscr{S}} ; \tau_{\mathscr{T}}=\sqrt{\boldsymbol{\sigma}_{\mathscr{S}}}(\mathscr{T}): \mathbf{c}_{\phi}^{-1}: \boldsymbol{\sigma}_{\mathscr{S}}(\mathscr{T})$ \\
\hline $\begin{array}{l}\text { Load.-unl. } \\
\text { conditions }\end{array}$ & $\bar{\gamma} \geqslant 0 \quad \bar{\phi} \leqslant 0 \quad \bar{\gamma} \bar{\phi}=0$ \\
\hline $\begin{array}{l}\text { Softening } \\
\text { rule }\end{array}$ & $\left\{\begin{array}{l}q_{\mathscr{S}} \in\left[0, q_{\mathrm{SD}}\right] \\
q_{\mathrm{SD}}=\left.q_{\mathscr{S}}\right|_{\mathrm{SD}}\end{array}\right.$ \\
\hline
\end{tabular}


1 Notice that, according to the previous reasonings, the discrete constitutive model (48) is implicitly induced from:

(1) the introduction of the softening regularization condition (39) into the continuum constitutive model (23),

(2) the presence of unbounded strains measures that develop at the discontinuous interface, according to Equation (7), and

(3) the imposition of the traction continuity Equation (14).

These are the only specific ingredients introduced by the SDA in addition to a standard continuum modelling. For the numerical simulation purposes the simulation is carried out in the continuum format on the basis of the continuum model (23) and the aforementioned

11 ingredients.

\section{FINITE ELEMENT APPROACH}

13 Conceptually there are not substantial differences between the finite element technology for the infinitesimal strain case, reported elsewhere [14], and the one used here for the numerical

15 simulations in large strain settings. Therefore, in this section only the outline of the theoretical foundations of considered finite element with embedded discontinuity will we provided,

17 emphasizing the specific features introduced by the large deformation kinematics.

\subsection{Discretized displacement field}

19 Let us consider the material domain $\Omega$ discretized in a triangular*** finite element mesh with $n_{\text {elem }}$ elements and $n_{\text {node }}$ nodes crossed by the discontinuity interface $\mathscr{S}$ (see Figure $6(\mathrm{a})$ ). Let

21 us then consider the subset $\mathscr{J}$ of the $n_{\mathscr{f}}$ elements that are crossed by $\mathscr{S}$ at the considered time $t$ :

$$
\mathscr{J} \equiv\left\{e \mid \Omega_{e} \cap \mathscr{S} \neq \emptyset\right\}=\left\{e_{i,}, \ldots, e_{m}, \ldots, e_{p}, \ldots\right\}
$$

This subset is determined by means of an specific algorithm devoted to track the discontinu-

25 ity [14]. For every element of $\mathscr{J}$, the tracking algorithm also provides the position of the elemental discontinuity interface $\mathscr{S}_{e}$ (see Figure 6(b)) of length $l_{e}$ which defines the domains

$27 \Omega_{e}^{+}$and $\Omega_{e}^{-}$and leaves one node at one side of the element (the solitary node $j^{\text {sol }}$ ) and two nodes $\left(j^{1}\right.$ and $\left.j^{2}\right)$ at the other side. The sense of the normal $\mathbf{N}$ inside the element is chosen

29 to point toward the solitary node side $\Omega_{e}^{+}$.

Based on this, and motivated by the kinematics presented in Section 2, we consider the 31 following interpolation of the (rate of) displacement field $\mathbf{u}^{(e)}$ inside a given element $e$ [15]:

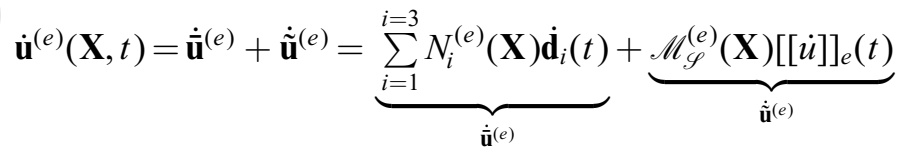

\footnotetext{
***From now on the three noded (constant stress) triangle will be considered as the basic element for explanation purposes. Generalization to other families of finite elements can also be done but it is out of the scope of this work.
} 


\section{NME 607}

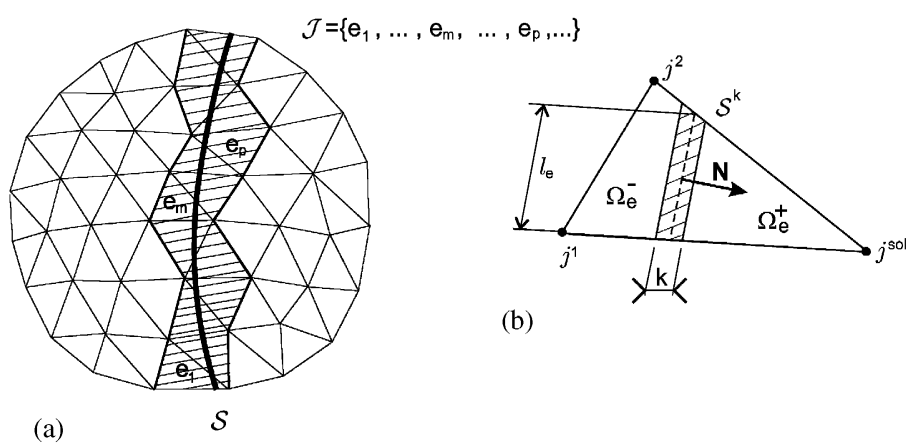

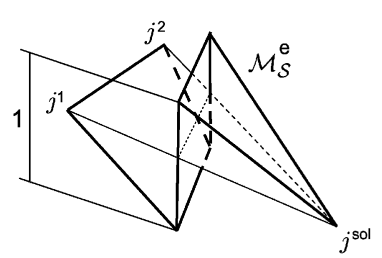

(c)

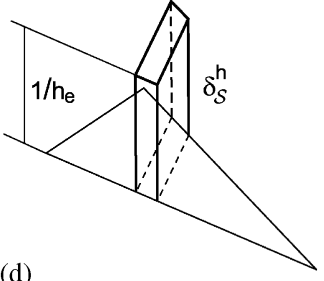

(d)

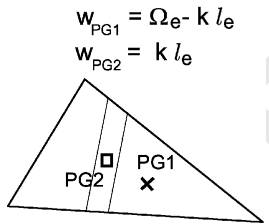

(e)

Figure 6. Finite element with embedded discontinuity.

1 where $\dot{\overline{\mathbf{u}}}^{(e)}$ is the standard $\mathscr{C}^{0}$ displacement field, interpolated by the shape functions $\left\{N_{1}^{(e)}\right.$, $\left.N_{2}^{(e)}, N_{3}^{(e)}\right\}$ of the linear isoparametric triangle [32], in terms of the nodal displacements $\mathbf{d}_{i}(t)$

3 at node $i$. The term $\dot{\tilde{\mathbf{u}}}$, in Equation (50), captures the singular (discontinuous) part of the displacement field in terms of the elemental displacement jump $[[\dot{\mathbf{u}}]]_{e}$ and the unit jump

5 function $\mathscr{M}_{\mathscr{S}}^{e}(\mathbf{X})$ defined as follows:

$$
\mathscr{M}_{\mathscr{S}}^{(e)}(\mathbf{X})= \begin{cases}0 & \forall e \notin \mathscr{J} \\ \mathscr{H}_{\mathscr{S}}^{(e)}(\mathbf{X})-N_{\mathrm{sol}}^{(e)}(\mathbf{X}) & \forall e \in \mathscr{J}\end{cases}
$$

7 where $\mathscr{H}_{\mathscr{S}}^{(e)}$ is the step function $\left(\mathscr{H}_{\mathscr{S}}^{(e)}(\mathbf{X})=1 \forall \mathbf{X} \in \Omega_{e}^{+}\right.$and $\left.\mathscr{H}_{\mathscr{S}}^{(e)}(\mathbf{X})=0 \forall \mathbf{X} \in \Omega_{e}^{-}\right)$and the index 'sol' refers to the solitary node. Figure $6(\mathrm{c})$ shows the $\mathscr{M}_{\mathscr{S}}^{(e)}$ function and emphasizes

9 its elemental support.

The term $\dot{\tilde{\mathbf{u}}}^{(e)}$ in Equation (50) can be regarded as an enhancement of the basic displacement 11 field $\dot{\overline{\mathbf{u}}}^{(e)}$, provided by the underlying isoparametric finite element, which due to the particular structure of the unit jump function $\mathscr{M}_{\mathscr{S}}^{e}$ in Equation (51) makes the resulting displacement 13 field discontinuous.

The kinematics of Equation (50) can be also expressed in compact form as

$$
\begin{aligned}
\dot{\mathbf{u}}(\mathbf{X}, t) & =\overline{\mathbf{N}}(\mathbf{X}) \cdot \dot{\mathbf{d}}(\mathbf{t})+\tilde{\mathbf{M}}(\mathbf{X}) \cdot \dot{\gamma}(\mathbf{t}) \\
\dot{\mathbf{d}} & \equiv\left\{\dot{\mathbf{d}}_{1}, \ldots, \dot{\mathbf{d}}_{n_{\text {node }}}\right\}^{\mathrm{T}} ; \dot{\gamma} \equiv\left\{[[\dot{\mathbf{u}}]]_{1}, \ldots,[[\dot{\mathbf{u}}]]_{n_{\mathcal{f}}}\right\}^{\mathrm{T}}
\end{aligned}
$$




\section{NME 607}

1 From Equations (50) and (51), the discrete (rate of) deformation gradient reads:

$$
\dot{\mathbf{F}}^{(e)}=\dot{\mathbf{u}}^{(e)} \otimes \nabla_{\mathbf{X}}=\underbrace{\sum_{i=1}^{i=3}\left(\dot{\mathbf{d}}_{i} \otimes \nabla_{\mathbf{X}} N_{i}^{(e)}\right)-\left([[\dot{\mathbf{u}}]]_{e} \otimes \nabla_{\mathbf{X}} N_{\text {sol }}^{(e)}\right)}_{\dot{\overline{\mathbf{F}}}^{(e)}{ }_{\text {bounded })}}+\delta_{\mathscr{S}}\left([[\dot{\mathbf{u}}]]_{e} \otimes \mathbf{N}\right)
$$

3 where $\delta_{\mathscr{S}}$ stands for Dirac's delta function emerging from the spatial derivation of the Heaviside function $\mathscr{H}_{\mathscr{S}}^{(e)}$ in Equation (51) $\left(\nabla_{\mathbf{X}} \mathscr{H}_{\mathscr{S}}^{(e)}(\mathbf{X})=\delta_{\mathscr{S}} \mathbf{N}\right)$. Notice that Equation (53) matches

5 the strong discontinuity kinematics discussed in Section 2.

As pointed out there, in order to overcome the numerical difficulties of treating with the

7 Dirac's delta function, and also to model the transition from the weak to the strong discontinuity regimes, $\delta_{\mathscr{S}}$ is replaced by a regularized function $\delta_{\mathscr{S}}^{(e)}$ defined within the element $e$ 9 as

$$
\delta_{\mathscr{S}}^{(e)}=\mu_{\mathscr{S}}^{(e)} \frac{1}{h_{e}}
$$

11 where $h_{e}$ is the elemental bandwidth, defined according the variable bandwidth model, and $\mu_{\mathscr{S}}^{(e)}$ is a collocation function whose support is the domain $\mathscr{S}_{e}^{k}$ in Figure 6(b) defined in terms

13 of the regularization parameter $k$ :

$$
\begin{array}{ll}
\mu_{\mathscr{S}}^{(e)}(\mathbf{X})=1 \quad \forall \mathbf{X} \in \mathscr{S}_{e}^{k} \\
\mu_{\mathscr{S}}^{(e)}(\mathbf{X})=0 & \forall \mathbf{X} \notin \mathscr{S}_{e}^{k}
\end{array}
$$

15 By considering Equations (54) and (55) the regularized form of the rate of deformation gradient (53) reads:

$$
\dot{\mathbf{F}}^{(e)}=\underbrace{\sum_{i=1}^{i=3}\left(\dot{\mathbf{d}}_{i} \otimes \nabla_{\mathbf{X}} N_{i}^{(e)}\right)-\left([[\dot{\mathbf{u}}]]_{e} \otimes \nabla_{\mathbf{X}} N_{\text {sol }}^{(e)}\right)}_{\dot{\mathbf{F}}^{(e)}{ }_{(\text {bounded })}}+\underbrace{\mu_{\mathscr{S}}^{(e)} \frac{1}{h_{e}}\left([[\dot{\mathbf{u}}]]_{e} \otimes \mathbf{N}\right)}_{\text {unbounded for } h_{e} \rightarrow 0}
$$

In order to integrate the discontinuous terms emerging from the second term of the right-

19 hand side of Equation (56), in addition to the regular sampling point of the constant strain triangle (PG1 in Figure 6(e)), the element is equipped with a second integration point (PG2

21 in Figure 6(e)) whose associated area is

$$
\operatorname{measure}\left(\mathscr{S}_{e}^{k}\right)=k l_{e}
$$

23 The regularization parameter $k$ has an arbitrary small value (as small as permitted by the machine precision). Therefore, integration of regular (bounded) terms in $\mathscr{S}_{e}^{k}$ results in

25 arbitrary small values, which makes the approach consistent. Also notice that neither $k$ nor $h_{e}$ are associated to any length of the finite element or mesh. 


\section{NME 607}

1 4.2. Body equilibrium and discrete equilibrium equations

Let us consider the weak form of the local equilibrium equations (13) in $\Omega \backslash \mathscr{S}$ :

$$
\delta \Pi_{\Omega \backslash \mathscr{S}}(\mathbf{u} ; \overline{\boldsymbol{\eta}})=\int_{\Omega \backslash \mathscr{S}} \mathbf{P}:\left(\overline{\boldsymbol{\eta}} \otimes \nabla_{\mathbf{X}}\right) \mathrm{d} \Omega-\int_{\Omega \backslash \mathscr{S}} \overline{\mathbf{B}} \cdot \overline{\boldsymbol{\eta}} \mathrm{d} \Omega-\int_{\Gamma_{\sigma}} \mathscr{T}^{\text {ext }} \cdot \overline{\boldsymbol{\eta}} \mathrm{d} \Gamma=\mathbf{0}
$$

$$
\forall \overline{\boldsymbol{\eta}} \in \overline{\mathscr{V}}_{0} \stackrel{\text { def }}{\equiv}\left\{\overline{\boldsymbol{\eta}}(\mathbf{X}) ;\left.\overline{\boldsymbol{\eta}}\right|_{\mathbf{X} \in \Gamma_{u}}=\mathbf{0}\right\}
$$

As previously stated, in Equation (14) an additional traction continuity condition should

5 be imposed in $\mathscr{S}$ to induce at this interface the discrete (traction vs displacement jump) constitutive equation. This reads:

$$
\mathbf{P}_{S} \cdot \mathbf{N}=\mathbf{P}_{\Omega^{+}} \cdot \mathbf{N} \quad\left(=\mathbf{P}_{\Omega^{-}} \cdot \mathbf{N}\right)=\mathscr{T} \quad \text { for }(\mathbf{X}, t) \in \mathscr{S} \times[0, T]
$$

After introducing the spatial discretization of Equation (52) the discrete counterpart of Equa9 tion (58) reads: ${ }^{\dagger \dagger \dagger}$

$$
\begin{aligned}
\delta \Pi_{\Omega}^{\mathbf{h}}\left(\mathbf{u}^{\mathbf{h}} ; \overline{\boldsymbol{\eta}}^{h}\right)=\int_{\Omega} \mathbf{P}:\left(\overline{\boldsymbol{\eta}}^{h} \otimes \nabla_{\mathbf{X}}\right) \mathrm{d} \Omega-\underbrace{\int_{\Omega} \overline{\mathbf{B}} \cdot \overline{\boldsymbol{\eta}}^{h} \mathrm{~d} \Omega-\int_{\Gamma_{\sigma}} \mathscr{T}^{\text {ext }} \cdot \overline{\boldsymbol{\eta}}^{h} \mathrm{~d} \Gamma}_{G^{\text {ext }}}=\mathbf{0} \\
\forall \overline{\boldsymbol{\eta}}^{h} \in \overline{\mathscr{V}}_{0}^{h}:=\overline{\boldsymbol{\eta}}^{h}(\mathbf{X})=\overline{\mathbf{N}} \cdot \boldsymbol{\delta} \mathbf{d} ;\left.\boldsymbol{\delta} \mathbf{d}\right|_{\boldsymbol{\Gamma}_{\mathbf{u}}}=\mathbf{0}
\end{aligned}
$$

11 On the other hand, the nominal traction continuity condition (59) can be weakly enforced in terms of the averages of $\mathscr{T}=\mathbf{P} \cdot \mathbf{N}$ inside every element $e \in \mathscr{J}$ as follows:

$$
\begin{aligned}
& \underbrace{\frac{1}{k l_{e}} \int_{\mathscr{P}_{e}^{k}} \mathbf{P} \cdot \mathbf{N} \mathrm{d} \Omega}_{\text {mean value of } \mathscr{T} \text { on } \mathscr{S}_{e}^{k}}=\underbrace{\frac{1}{\Omega_{e}} \int_{\Omega_{e}} \mathbf{P} \cdot \mathbf{N d} \Omega}_{\text {mean value of } \mathscr{T} \text { on } \Omega_{e}} \forall e \in \mathscr{J} \Rightarrow \\
& \int_{\Omega_{e}}\left(\mu_{\mathscr{S}}^{(e)} \frac{1}{k l_{e}}-\frac{1}{\Omega_{e}}\right) \mathbf{P} \cdot \mathbf{N} \mathrm{d} \Omega=\mathbf{0} \quad \forall e \in \mathscr{J}
\end{aligned}
$$

13 where the discontinuous character of the function $\mu_{\mathscr{S}}^{(e)}$ inside the element (see Equation (55)) can be captured by the integration rule sketched in Figure 6(e).

Finally, some algebraic manipulation of Equations (60) and (62) leads to:

$$
\begin{aligned}
\int_{\Omega_{i}} \mathbf{P} \cdot\left(\nabla_{\mathbf{X}} N_{i}\right) \mathrm{d} \Omega-\mathbf{f}_{i}^{\mathrm{ext}} & =\mathbf{0} \quad \forall i \in\left\{1, \ldots, n_{\text {node }}\right\} \\
\int_{\Omega_{e}}\left(\mu_{\mathscr{S}}^{(e)} \frac{1}{k}-\frac{l_{e}}{\Omega_{e}}\right) \mathbf{P} \cdot \mathbf{N} \mathrm{d} \Omega & =\mathbf{0} \quad \forall e \in \mathscr{J} \\
\mathbf{f}_{i}^{\mathrm{ext}} & =\int_{\Omega_{i}} N_{i} \overline{\mathbf{B}} \mathrm{d} V+\int_{\partial \Omega_{i} \cap \Gamma_{\sigma}} N_{i} \mathscr{T}^{\mathrm{ext}} \mathrm{d} \Gamma
\end{aligned}
$$

$\overline{t^{\dagger} \text { Observe that, }}$ due to the zero measure of the interface $\mathscr{S}$ and the bounded character of the integral kernels, the integration domain can be extended from $\Omega \backslash \mathscr{S}$ to $\Omega$. 


\section{NME 607}

1 where $\Omega_{i}$ stands for the support of the shape function $N_{i}$. The discrete system of Equations (63) provides a set of $n_{\text {node }}+n_{\mathscr{f}}$ non-linear equations to solve for the $n_{\text {node }}+n_{\mathscr{f}}$ un-

3 knowns $\mathbf{d} \equiv\left\{\mathbf{d}_{1}, \ldots, \mathbf{d}_{n_{\text {node }}}\right\} ; \gamma \equiv\left\{[[\mathbf{u}]]_{1}, \ldots,[[\mathbf{u}]]_{n_{\mathcal{f}}}\right\}$ of the discrete problem as pointed out in Equation (52).

5 For computational purposes, and since the constitutive equations are given in terms of the symmetric Kirchhoff stresses $\tau=\mathbf{P} \cdot \mathbf{F}^{\mathrm{T}}$, Equation (63) can be appropriately rewritten, taking

7 into account the identity $\nabla_{\mathbf{X}}(\bullet)=\nabla_{\mathbf{X}}(\bullet) \cdot \mathbf{F}$, as

$$
\begin{array}{ll}
\int_{\Omega_{i}} \tau \cdot\left(\nabla_{\mathbf{x}} N_{i}\right) \mathrm{d} \Omega-\mathbf{f}_{i}^{\text {ext }}=\mathbf{0} & \forall i \in\left\{1, \ldots, n_{\text {node }}\right\} \\
\int_{\Omega_{e}}\left(\mu_{\mathscr{S}}^{(e)} \frac{1}{k}-\frac{l_{e}}{\Omega_{e}}\right) \tau \cdot \mathbf{n} \mathrm{d} \Omega=\mathbf{0} & \forall e \in \mathscr{J}
\end{array}
$$

9 where $\mathbf{n}=\mathbf{F}^{-\mathrm{T}} \cdot \mathbf{N}$ is the convected normal at the spatial configuration in Equation (12), and $(\bullet)^{\mathrm{T}}$ stands for the transpose of $(\bullet)$. For the considered 2D case in a cartesian $(x, y)$ co-ordinate

11 system, Equation (65) can then be cast into the classical B-matrix format [32] as

$$
\bigcup_{e=1}^{e=n_{\text {clem }}}\left[\int_{\Omega_{e}} \mathbf{B}^{(e)^{\mathrm{T}}} \cdot\{\boldsymbol{\tau}\} \quad \mathrm{d} \Omega-\mathbf{F}^{\operatorname{ext}(e)}\right]=\mathbf{0}
$$

where $\cup$ stands for the assembling operator and the elemental $B$-matrix, $\mathbf{B}^{(e)}$, the $2 \mathrm{D}$ Kirchhoff 15 stress vector $\{\tau\}$, and nodal forces vector, $\mathbf{F}^{\mathrm{ext}(e)}$, are given by

$$
\begin{aligned}
& \mathbf{B}^{(e)}=\left[\begin{array}{llll}
\mathbf{B}_{1}^{(e)} & \mathbf{B}_{2}^{(e)} & \mathbf{B}_{3}^{(e)} & \mathbf{G}^{(e)}
\end{array}\right] \\
& \mathbf{B}_{i}^{(e)}=\left[\begin{array}{cc}
\partial_{x} N_{i}^{(e)} & 0 \\
0 & \partial_{y} N_{i}^{(e)} \\
\partial_{y} N_{i}^{(e)} & \partial_{x} N_{i}^{(e)}
\end{array}\right] ; \quad \mathbf{G}^{(e)}=\left(\mu_{\mathscr{S}}^{(e)} \frac{1}{k}-\frac{l_{e}}{\Omega_{e}}\right)\left[\begin{array}{cc}
n_{x} & 0 \\
0 & n_{y} \\
n_{y} & n_{x}
\end{array}\right] \\
& \{\boldsymbol{\tau}\}=\left[\begin{array}{c}
\tau_{x x} \\
\tau_{y y} \\
\tau_{x y}
\end{array}\right] \quad \mathbf{n}=\left[\begin{array}{c}
n_{x} \\
n_{x}
\end{array}\right] ; \quad \mathbf{F}^{\operatorname{ext}(e)}=\left[\begin{array}{c}
\mathbf{f}_{1}^{\operatorname{ext}(e)} \\
\mathbf{f}_{2}^{\operatorname{ext}(e)} \\
\mathbf{f}_{3}^{\operatorname{ext}(e)} \\
\mathbf{0}
\end{array}\right]
\end{aligned}
$$

The structure of Equations (67) and (68) suggests the introduction of an internal additional

17 fourth node for each element $e$, that is activated only for the elements crossed by the discontinuity interface $(e \in \mathscr{J})$ and whose corresponding degrees of freedom and associated shape

19 function are, respectively, the displacement jumps $[[\mathbf{u}]]_{e}$ and $\mathscr{M}_{\mathscr{S}}^{(e)}$ in Equations (50) and (51). Since the support of $\mathscr{M}_{\mathscr{S}}^{(e)}$ is only $\Omega_{e}$, those internal degrees of freedom can be eventually condensed at the elemental level and removed from the global system of equations. 


\section{NME 607}

THE STRONG DISCONTINUITY APPROACH

\section{4.3. Time integration and linearization}

In the context of a time advancing process, the rate equation (56), within the element $(e)$, is

3 integrated at time $t+\Delta t$, in terms of the corresponding values at time $t$ and the incremental values of the nodal unknowns $\Delta \mathbf{d}_{i}$ and $\Delta[[\mathbf{u}]]_{e}$, as follows: ${ }^{\ddagger \ddagger}$

$$
\begin{aligned}
\mathbf{F}(\mathbf{X}, t+\Delta t) \stackrel{\text { not }}{=} & \mathbf{F}_{t+\Delta t} \\
= & \mathbf{F}_{t}+\sum_{i=1}^{i=3}\left[\Delta \mathbf{d}_{i} \otimes \nabla_{\mathbf{X}} N_{i}^{(e)}\right]-\left(\Delta\left[\left[\mathbf{u}_{e}\right]\right] \otimes \nabla_{\mathbf{X}} N_{\text {sol }}^{(e)}\right) \\
& +\mu_{\mathscr{S}}^{(e)} \frac{1}{h_{e}(t)}\left(\Delta[[\mathbf{u}]]_{e} \otimes \mathbf{N}\right) \\
\Delta \mathbf{d}_{i}= & \mathbf{d}_{i}(t+\Delta t)-\mathbf{d}_{i}(t) \\
\Delta[[\mathbf{u}]]_{e}= & {[[\mathbf{u}]]_{e}(t+\Delta t)-[[\mathbf{u}]]_{e}(t) }
\end{aligned}
$$

5 On the other hand, the algorithm of the continuum constitutive model updates the stresses $\tau_{t+\Delta t}$, in terms of the updated gradient of deformation tensor $\mathbf{F}_{t+\Delta t}$ and the previous values

7 of the stresses $\tau_{t}$, and the internal variables $q_{t}$, and also provides the algorithmic constitutive operator $\mathbf{c}_{t+\Delta t}^{\text {tang }}$ (see the appendix):

$$
\tau_{t+\Delta t}=\mathscr{F}\left(\mathbf{F}_{t+\Delta t}, \tau_{t}, q_{t}\right) ; \quad L_{\overline{\mathbf{v}}} \tau_{t+\Delta t}=\mathbf{c}_{t+\Delta t}^{\mathrm{tang}}:\left(\nabla_{\mathbf{x}} \otimes \dot{\mathbf{u}}_{t+\Delta t}\right)^{S}
$$

Using standard procedures [33], linearization, in the direction $\dot{\mathbf{u}}_{t+\Delta t}$, of the equilibrium equa11 tions (60) and (62) at time $t+\Delta t$ yields:

$$
\begin{array}{cc}
\int_{\Omega_{i}}\left(\overline{\boldsymbol{\eta}}^{h} \otimes \nabla_{\mathbf{x}}\right):\left[\left(\dot{\mathbf{u}}_{t+\Delta t} \otimes \nabla_{\mathbf{x}}\right) \cdot \tau_{t+\Delta t}+L_{\overline{\mathbf{v}}}\left(\tau_{t+\Delta t}\right)\right] \mathrm{d} \Omega-\dot{G}^{\mathrm{ext}}=0 & \forall \overline{\boldsymbol{\eta}}^{h} \in \overline{\mathscr{V}}_{0}^{h} \\
\int_{\Omega_{e}}\left(\mu_{\mathscr{S}}^{(e)} \frac{1}{k}-\frac{l_{e}}{\Omega_{e}}\right)\left(\left(\dot{\mathbf{u}}_{t+\Delta t} \otimes \nabla_{\mathbf{x}}\right) \cdot \tau_{t+\Delta t} \cdot \mathbf{n}+L_{\overline{\mathbf{v}}}\left(\tau_{t+\Delta t}\right) \cdot \mathbf{n}\right) \mathrm{d} \Omega=0 & \forall e \in \mathscr{J}
\end{array}
$$

13 which, after substitution of Equation (70) and some algebraic manipulation, reads:

$$
\begin{array}{cc}
\int_{\Omega_{i}}\left(\overline{\boldsymbol{\eta}}^{h} \otimes \nabla_{\mathbf{x}}\right):\left[\left(\mathbb{1} \otimes \boldsymbol{\tau}_{t+\Delta t}\right)+\mathbf{c}_{t+\Delta t}^{\mathrm{tang}}\right]:\left(\nabla_{\mathbf{x}} \otimes \dot{\mathbf{u}}_{t+\Delta t}\right) \mathrm{d} \Omega-\dot{G}^{\mathrm{ext}}=0 & \forall \overline{\boldsymbol{\eta}}^{h} \in \overline{\mathscr{V}}_{0}^{h} \\
\int_{\Omega_{e}}\left(\mu_{\mathscr{S}}^{(e)} \frac{1}{k} m-\frac{l_{e}}{\Omega_{e}}\right) \mathbf{n} \cdot\left[\left(\tau_{t+\Delta t} \otimes \mathbb{1}\right)+\mathbf{c}_{t+\Delta t}^{\mathrm{tang}}\right]:\left(\nabla_{\mathbf{x}} \otimes \dot{\mathbf{u}}_{t+\Delta t}\right) \mathrm{d} \Omega=0 & \forall e \in \mathscr{J}
\end{array}
$$

$¥ \ddagger$ As a technical detail in Equation (69) notice that the elemental bandwidth is updated with one time step delay $\left(h_{e_{t+\Delta t}} \equiv h_{e}(t)\right)$. In the context of the variable bandwidth method at the weak discontinuity regime, this explicit update makes linear that equation, with a considerable simplification of the whole procedure while keeping the consistency of the integration method. 


\section{NME 607}

1 were $\left(\mathbb{1} \bar{\otimes} \tau_{t+\Delta t}\right)_{i j k l} \stackrel{\text { def }}{=} \delta_{i k} \tau_{j l}$ and $\left(\tau_{t+\Delta t} \otimes \mathbb{1}\right)_{i j k l}=\tau_{i l} \delta_{j k}$. From Equations (50), (51) and (60), the terms $\nabla_{\mathbf{x}} \otimes \dot{\mathbf{u}}_{t+\Delta t}$ and $\left(\overline{\boldsymbol{\eta}}^{h} \otimes \boldsymbol{\nabla}_{\mathbf{x}}\right)$ in Equation (71) can be expressed in discrete form as:

$$
\begin{aligned}
\nabla_{\mathbf{x}} \otimes \dot{\mathbf{u}}_{t+\Delta t} & =\sum_{i=1}^{i=3} \nabla_{\mathbf{x}} N_{i}^{(e)} \otimes \dot{\mathbf{d}}_{i_{t+\Delta t}}+\mu_{\mathscr{S}}^{(e)} \frac{1}{h_{e}(t)} \mathbf{n} \otimes[[\dot{\mathbf{u}}]]_{e_{t+\Delta t}}-\nabla_{\mathbf{x}} N_{\mathrm{sol}}^{(e)} \otimes[[\dot{\mathbf{u}}]]_{e_{t+\Delta t}} \\
\overline{\boldsymbol{\eta}}^{h} \otimes \nabla_{\mathbf{x}} & =\sum_{i=1}^{i=3} \delta \mathbf{d} \otimes \nabla_{\mathbf{x}} N_{i}^{(e)}
\end{aligned}
$$

After insertion of Equation (73) and some algebraic manipulations Equation (72) can be 5 rewritten, in discrete form and for the $2 \mathrm{D}$ problem in a cartesian $(x, y)$ co-ordinate system, in the following B-matrix format:

7

$$
\bigcup_{e=1}^{e=n_{\text {elem }}} \underbrace{\left[\int_{\Omega_{e}} \mathbf{B}_{\text {geo }}^{(e)^{\mathrm{T}}} \cdot\left[\hat{\tau}_{t+\Delta t}\right] \cdot \mathbf{B}_{\mathrm{geo}}^{*(e)} \mathrm{d} \Omega\right.}_{\mathbf{K}_{\text {geo }}}+\underbrace{\left.\int_{\Omega_{e}} \mathbf{B}^{(e)^{\mathrm{T}}} \cdot \mathbf{c}_{t+\Delta t}^{\mathrm{tang}} \cdot \mathbf{B}^{*(e)} \mathrm{d} \Omega\right]}_{\mathbf{K}_{\mathrm{mat}}} \cdot\left[\begin{array}{c}
\mathbf{d}^{(e)} \\
{[[\dot{\mathbf{u}}]]_{e}}
\end{array}\right]_{t+\Delta t}=\dot{\mathbf{F}}_{t+\Delta t}^{\mathrm{ext}(e)}
$$

where $\mathbf{F}^{\text {ext } e}$ is given in Equation (68) and $\mathbf{K}_{\text {geo }}$ and $\mathbf{K}_{\text {mat }}$ can be recognized, respectively, as the

9 classical geometrical and material tangent stiffness [32]. The remaining terms of Equation (74) can be described as:

$$
\begin{aligned}
& \mathbf{B}^{(e)}=\left[\mathbf{B}_{1}^{(e)}, \mathbf{B}_{2}^{(e)}, \mathbf{B}_{3}^{(e)}, \mathbf{G}^{(e)}\right] \\
& \mathbf{B}^{*(e)}=\left[\mathbf{B}_{1}^{(e)}, \mathbf{B}_{2}^{(e)}, \mathbf{B}_{3}^{(e)}, \mathbf{G}_{t+\Delta t}^{*^{(e)}}\right] \\
& \mathbf{B}_{i}^{(e)}=\left[\begin{array}{cc}
\partial_{x} N_{i}^{(e)} & 0 \\
0 & \partial_{y} N_{i}^{(e)} \\
\partial_{y} N_{i}^{(e)} & \partial_{x} N_{i}^{(e)}
\end{array}\right] \\
& \mathbf{G}^{(e)}=\left(\mu_{\mathscr{S}}^{(e)} \frac{1}{k}-\frac{l_{e}}{\Omega_{e}}\right)\left[\begin{array}{cc}
n_{x} & 0 \\
0 & n_{y} \\
n_{y} & n_{x}
\end{array}\right] \\
& \mathbf{G}_{t+\Delta t}^{*^{(e)}}=\mu_{\mathscr{S}}^{(e)} \frac{1}{h_{e}(t)}\left[\begin{array}{cc}
n_{x} & 0 \\
0 & n_{y} \\
n_{y} & n_{x}
\end{array}\right]-\left[\begin{array}{cc}
\partial_{x} N_{\mathrm{sol}}^{(e)} & 0 \\
0 & \partial_{y} N_{\mathrm{sol}}^{(e)} \\
\partial_{y} N_{\mathrm{sol}}^{(e)} & \partial_{x} N_{\mathrm{sol}}^{(e)}
\end{array}\right] \\
& \mathbf{B}_{\mathrm{geo}^{(e)}}^{(e)}\left[\mathbf{B}_{\mathrm{geo}_{1}}^{(e)}, \mathbf{B}_{\mathrm{geO}_{2}}^{(e)}, \mathbf{B}_{\mathrm{geo}_{3}}^{(e)}, \mathbf{G}_{\mathrm{geo}^{(e)}}^{(e)}\right. \\
& \mathbf{B}_{\mathrm{geo}^{(e)}}^{*(e)}=\left[\mathbf{B}_{\mathrm{geo}_{1}}^{(e)}, \mathbf{B}_{\mathrm{geO}_{2}}^{(e)}, \mathbf{B}_{\mathrm{geo}_{3}}^{(e)}, \mathbf{G}_{\mathrm{geo}_{t+\Delta t}}^{*(e)}\right]
\end{aligned}
$$




$$
\begin{aligned}
& \mathbf{B}_{\text {geo }_{i}}^{(e)}=\left[\begin{array}{cc}
\partial_{x} N_{i}^{(e)} & 0 \\
\partial_{y} N_{i}^{(e)} & 0 \\
0 & \partial_{x} N_{i}^{(e)} \\
0 & \partial_{y} N_{i}^{(e)}
\end{array}\right] \\
& \mathbf{G}_{\mathrm{geo}}^{(e)}=\left(\mu_{\mathscr{S}}^{(e)} \frac{1}{k}-\frac{l_{e}}{\Omega_{e}}\right)\left[\begin{array}{cc}
n_{x}^{(e)} & 0 \\
n_{y}^{(e)} & 0 \\
0 & n_{x}^{(e)} \\
0 & n_{y}^{(e)}
\end{array}\right] \\
& \mathbf{G}_{\mathrm{geo}_{t+\Delta t}^{(e)}}^{\text {en }^{(e)}}=\mu_{\mathscr{S}}^{(e)} \frac{1}{h_{e}(t)}\left[\begin{array}{cc}
n_{x}^{(e)} & 0 \\
n_{y}^{(e)} & 0 \\
0 & n_{x}^{(e)} \\
0 & n_{y}^{(e)}
\end{array}\right]-\left[\begin{array}{cc}
\partial_{x} N_{\mathrm{sol}}^{(e)} & 0 \\
\partial_{y} N_{\mathrm{sol}}^{(e)} & 0 \\
0 & \partial_{x} N_{\mathrm{sol}}^{(e)} \\
0 & \partial_{y} N_{\mathrm{sol}}^{(e)}
\end{array}\right] \\
& \hat{\tau}=\left[\begin{array}{cccc}
\tau_{x x} & \tau_{x y} & 0 & 0 \\
\tau_{x y} & \tau_{y y} & 0 & 0 \\
0 & 0 & \tau_{x x} & \tau_{x y} \\
0 & 0 & \tau_{x y} & \tau_{y y}
\end{array}\right] \\
& \left\{\bar{L}_{\overline{\mathbf{v}}} \tau\right\}=\left[\begin{array}{c}
\left(\bar{L}_{\overline{\mathbf{v}}} \tau\right)_{x x} \\
\left(\bar{L}_{\overline{\mathbf{v}}} \tau\right)_{y y} \\
\left(\bar{L}_{\overline{\mathbf{v}}} \tau\right)_{x y}
\end{array}\right] ; \quad\left\{\nabla_{\mathbf{x}} \otimes \dot{\mathbf{u}}_{t+\Delta t}\right\}=\left[\begin{array}{c}
\partial_{x} \dot{u}_{x} \\
\partial_{y} \dot{u}_{y} \\
\partial_{y} \dot{u}_{x}+\partial_{x y} \dot{u}_{y}
\end{array}\right]_{t+\Delta t} \\
& \bar{L}_{\overline{\mathbf{v}}} \tau_{t+\Delta t}=\mathbf{c}_{t+\Delta t}^{\operatorname{tang}}:\left(\nabla_{\mathbf{x}} \otimes \dot{\mathbf{u}}_{t+\Delta t}\right)^{S} \Leftrightarrow\left\{\bar{L}_{\overline{\mathbf{v}}} \tau_{t+\Delta t}\right\}=\mathbf{c}_{t+\Delta t}^{\operatorname{tang}} \cdot\left\{\nabla_{\mathbf{x}} \otimes \dot{\mathbf{u}}_{t+\Delta t}\right\}
\end{aligned}
$$

1 Observe that, due to the differences $\mathbf{B}^{(e)} \neq \mathbf{B}^{*(e)}$ and $\mathbf{B}_{\text {geo }}^{(e)} \neq \mathbf{B}_{\text {geo }}^{*(e)}$ (emerging from the different matrices $\mathbf{G}^{(e)} \neq \mathbf{G}^{*(e)}$ and $\mathbf{G}_{\text {geo }}^{(e)} \neq \mathbf{G}_{\text {geo }}^{(e)}$ ) in Equations (75) and (76), the tangent stiffness

$3 \mathbf{K}=\mathbf{K}_{\text {geo }}+\mathbf{K}_{\text {mat }}$, in Equation (74), is not symmetric. This should be expected from the continuum formulation of the problem since the traction continuity equation (59) has not been

5 imposed from the variational principle (58), but enforced in an average or weighting procedure through Equation (61). This fact confers to the presented finite element procedure the character

7 of a Petrov-Galerkin finite element approximation in front of the classical Galerkin-based finite element approaches. The resulting procedure has been sometimes termed, in infinites-

9 imal strain settings, the Statically and Kinematically Optimal Non-symmetric formulation [34], emphasizing its improved behaviour in front of other symmetric alternative finite elements with embedded discontinuities. 


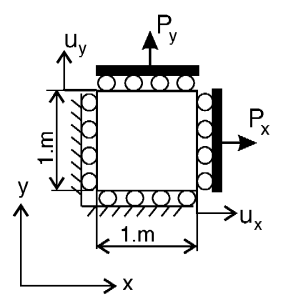

(a)

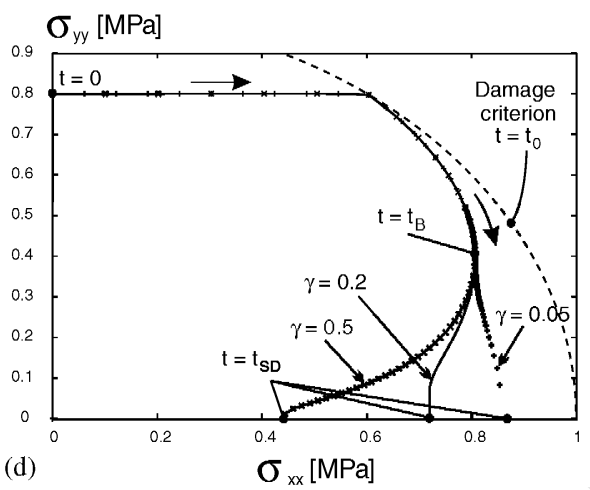

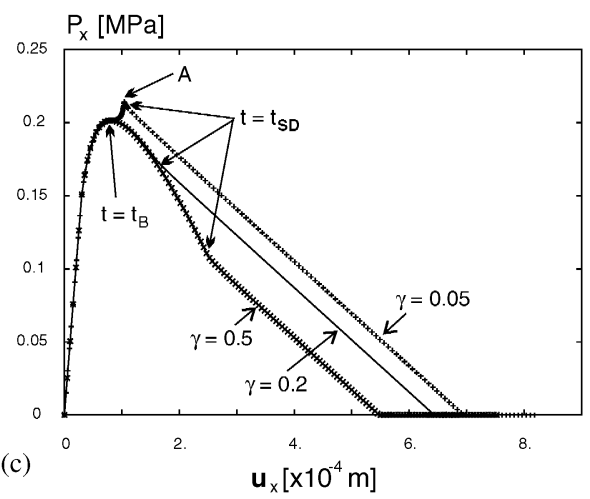

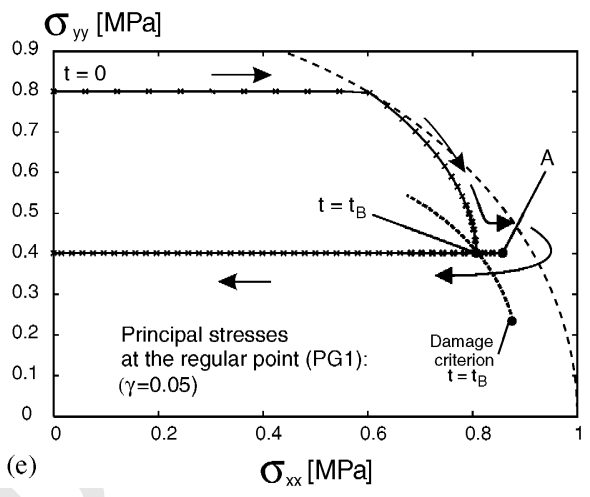

Figure 7. (a) Boundary conditions; (b) bandwidth variation law $h$ vs $q$; (c) total load $P_{x}$ vs lateral displacement $u_{x}$; (d) equilibrium path in the principal stress plane for a point in $\mathscr{S}$ (singular Gauss point

PG2 in Figure 1(e); and (e) idem for a point in $\Omega \backslash \mathscr{S}$ (regular Gauss point PG1 in Figure 1(e)).

\section{NUMERICAL SIMULATIONS}

In this section the numerical method described above is applied to the simulation of different

problems where strong discontinuities develop. The main goal is to show that these numerical simulations behave as predicted by the theoretical analyses, as well as to highlight the role

5 of large strain kinematics in the obtained results.

The constitutive model considered in the simulations is the continuum isotropic damage 7 model described in Equation (23) with the softening regularization condition (39). Therefore, it is expected that the discrete damage model (48) is induced at the interface of discontinuity

9 and the results to be the same than if this discrete model had explicitly been implemented.

\subsection{Specimen under biaxial stress state}

11 This example highlights the role of the variable bandwidth model in the presented approach. A square specimen is subjected to a biaxial stress state by imposing a constant displacement

$13 u_{y}$ and a gradually increasing displacement $u_{x}$ on the upper and right edges of the plate, respectively, see Figure 7(a). 
1 The material is characterized by the following data: elastic Lame's parameters $\lambda=0.0(\mathrm{MPa})$, $\mu=E / 2=1.10^{4}(\mathrm{MPa})$, continuum softening modulus $\mathscr{H}=-0.125$, discrete softening modulus $3 \quad \overline{\mathscr{H}}=-0.125\left(\mathrm{~cm}^{-1}\right)$.

The damage criterion is that defined in the appendix, with $\mu_{\phi}=\mu$ and $\lambda_{\phi}=\lambda$. In this circum-

5 stances the elastic threshold results $r_{0}=q_{0}=\sigma_{u} / \sqrt{E}=0.00707(\mathrm{MPa})^{1 / 2}$ (where $\sigma_{u}=1.0(\mathrm{MPa})$ stands for the uniaxial strength and $E=2.10^{4}(\mathrm{MPa})$ for Young modulus).

7 The bifurcation analysis determines the normal to the discontinuity interface as $\mathbf{e}_{n}=(1,0)^{\mathrm{T}}$ and the only non-trivial strong discontinuity condition in Equation (43) is $\sigma_{x x} \sigma_{y y}-\sigma_{x y}^{2}=0$.

9 Since, due to the geometrical symmetries and loading conditions, $\sigma_{x y}=0$, the strong discontinuity condition reads $\sigma_{y y}=0$ which clearly is not trivially fulfilled at the bifurcation time $t_{\mathrm{B}}$.

11 Therefore, bifurcation takes place under the form of a weak discontinuity, and a weak-strong discontinuity transition regime has to be introduced. This is governed by a variable bandwidth

13 law $h(q)$, given by (see Figure 7(b)):

$$
\begin{array}{ll}
h=h_{0}=1 \mathrm{~cm} ; & t \leqslant t_{\mathrm{B}}\left(q>q_{\mathrm{B}}\right) \\
h=k+\frac{h_{0}-k}{q_{\mathrm{B}}-q_{\mathrm{SD}}}\left(q-q_{\mathrm{SD}}\right) ; & t_{\mathrm{B}}<t<t_{\mathrm{SD}}\left(q_{\mathrm{SD}}<q<q_{\mathrm{B}}\right) \\
h=k ; & t \geqslant t_{\mathrm{SD}} \quad\left(q \leqslant q_{\mathrm{SD}}\right)
\end{array}
$$

15 where $q_{\mathrm{B}}$ and with $q_{\mathrm{SD}}$ stand for the values of the internal variable $q$ at the bifurcation time, $t_{\mathrm{B}}$, and at the strong discontinuity time, $t_{\mathrm{SD}}$, respectively. The value $q_{\mathrm{SD}}$ is defined

17 as $q_{\mathrm{SD}}=(1-\gamma) q_{\mathrm{B}}(\gamma \in[0,1])$. Therefore, the transition factor $\gamma$ determines the size of the weak discontinuity interval $\left[q_{\mathrm{SD}}, q_{\mathrm{B}}\right]$ so that for $\gamma=0$ there is no weak discontinuity regime

$19\left(q_{\mathrm{SD}}=q_{\mathrm{B}}\right)$ and the kinematics immediately after the bifurcation is imposed to be the strong discontinuity one. On the other hand, if $\gamma=1$ then $q_{\mathrm{SD}}=0$ and all the post-bifurcation stage

21 will be traced as a weak discontinuity.

As a matter of example, results, obtained with several values of $\gamma$, are presented in Figures 7(c) and 7(d).

- For a very short transition regime, ${ }^{\S \S}$ determined by a very small transition factor $\gamma=0.05$, it appears an unexpected artificial elastic loading (in terms of $P_{x}-u_{x}$ response) immediately after bifurcation (see point $\mathrm{A}$ in Figure 7(c)) followed by the regular expected unloading response. This can be explained as follows: since after bifurcation an incrementally elastic behaviour is algorithmically imposed at $\Omega \backslash S$, as expected from the theoretical bifurcation analysis, violation of the strong discontinuity conditions make the stresses at that point infringe the damage criterion as the process evolution proceeds (see Figure 7(e)). This results in an artificial elastic loading at that part of the body ${ }^{\text {Tी }}$ ( responsible, in turn, for the behaviour observed in Figure 7(c) up to point A, where the strong discontinuity condition $\sigma_{y y}=0$ is fulfilled at $\mathscr{S}$ (see Figure 7(d)). Beyond that point the strong discontinuity regime takes place and regular elastic unloading occurs at $\Omega \backslash \mathscr{S}$ (see Figure $7(\mathrm{e}))$ resulting in the $P_{x}-u_{x}$ unloading branch in Figure 7(c). There we can notice that:

\footnotetext{
$\S \S \S$ For practical purposes this is equivalent to enforce bifurcation into a strong discontinuity.

ฯ ฯ As a matter of fact if this artificial elastic loading takes place, a 'two material' constitutive equation (elastic at $\Omega \backslash \mathscr{S}$ and elasto plastic at $\mathscr{S}$ ) is artificially imposed by the algorithm.
} 


\section{NME 607}

- For longer (slower) transitions, determined for instance by $\gamma=0.2$ or $\gamma=0.5$, this artificial elastic loading is no longer observed and the transition from bifurcation to the strong discontinuity regime takes place smoothly as shown in Figure 7(c) and keeping the theoretical elastic unloading at $\Omega \backslash \mathscr{S}$.

These results confirm that, as predicted by the theoretical analyses, the strong discontinuity kinematics cannot be imposed, in general, immediately (or very shortly) after bifurcation since the strong discontinuity conditions (43) are not fulfilled at this time. Therefore, the transition regime (weak discontinuity) appears as a mechanism to smoothly induce these

9 strong discontinuity conditions preserving the bounded character of the stress and rate of stress fields.

11 In addition, it can be observed in Figure 7(c) that the final slopes of the $P_{x}-u_{x}$ curves are the same in all cases. This could have been expected from the fact that this part of the

13 structural response is ruled by the induced discrete (traction-displacement jump) constitutive equation which is independent of the size of the transition regime.

\section{5.2. Debinding problem: crack propagation in mode I}

This example is devoted to get some insight on the influence, on the response provided by the

17 SDA, of the chosen kinematics (large or infinitesimal strains). For comparison purposes the results using the infinitesimal strain counterpart of the continuum model (23) and the SDA

19 for infinitesimal strains settings given in [17] are used.

The induced discrete constitutive models for both cases (infinitesimal and large strains) are

21 made equivalent in terms of the fracture energy as a material property. The fracture energy $G_{\mathrm{f}}$, defined as the external mechanical energy required per unit of surface of the discontinuity

23 interface $\mathscr{S}$ to produce the total decohesion of the material [26], can be then computed as

$$
G_{\mathrm{f}}=\int_{t_{\mathrm{SD}}}^{t_{\infty}} \mathscr{T}(\mathbf{X}, \mathbf{t}) \cdot[[\dot{\mathbf{u}}]](\mathbf{X}, \mathbf{t}) \mathrm{d} t
$$

where it is assumed that complete decohesion $(\mathscr{T}=\mathbf{0})$ is achieved at time $t_{\infty}$.

Considering the same reference problem (uniaxial stress process) $G_{\mathrm{f}}$ can be computed and equated for both cases leading to:

$$
\begin{gathered}
\text { Small strains } G_{\mathrm{f}}=-\sigma_{\mathrm{u}}^{2} /\left(2 E \overline{\mathscr{H}}^{\text {small }}\right) \\
\text { Large strains } G_{\mathrm{f}}=-\sigma_{u}^{2} /\left(E \overline{\mathscr{H}}^{\text {large }}\right)
\end{gathered} \Rightarrow \overline{\mathscr{H}}^{\text {large }}=2 \overline{\mathscr{H}}^{\text {small }}
$$

29 where $\sigma_{\mathrm{u}}$ stands for the uniaxial peak stress and $E$ for the Young modulus. The relationship between the discrete softening modulus $\overline{\mathscr{H}}$, obtained in Equation (80), is then extended to

31 more general stress states as an approximate way to keep the fracture energy as a common material property for large and small strain kinematics.

33 With these considerations in mind, in Figure 8 the simulation of a debinding process in a composite panel is presented.

35 Two plates, initially bound together, are enforced to separate by pulling the upper notch, as depicted in Figure 8(a). Both the plates and the binding material are assumed to have the

37 same material properties, and, as a result of the loading process, a crack propagates vertically beneath the notch and along the binding. 


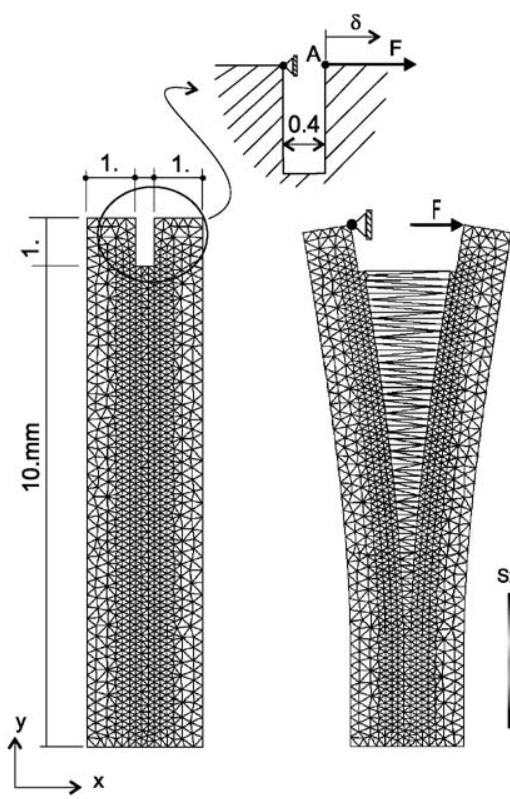

(a)

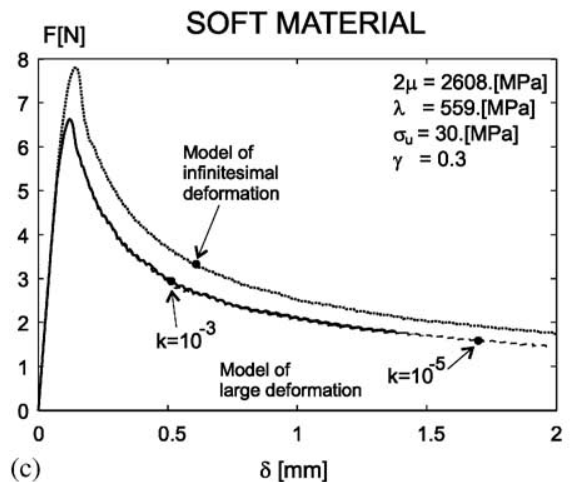

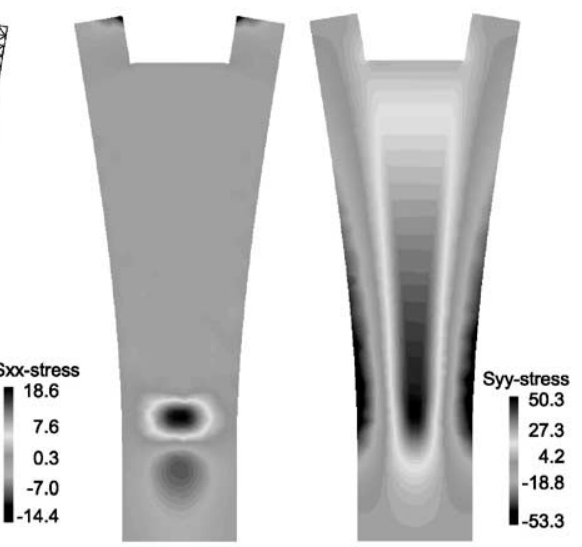

(b)

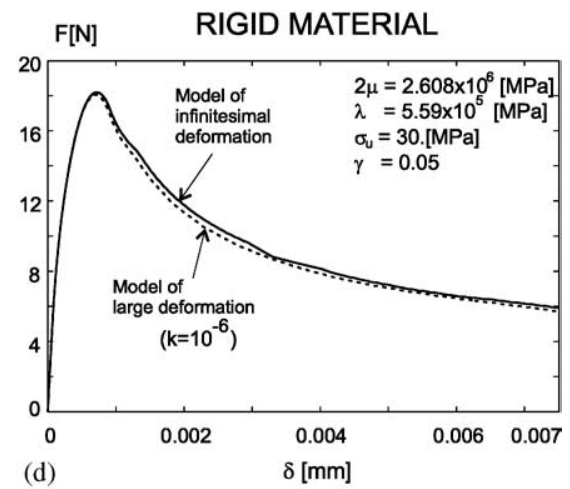

Figure 8. Crack propagation in Mode I: (a) geometry, boundary conditions and finite element mesh; (b) contours at the final time with the large deformation model: (deformed shape at true scale), Contours of the Cauchy stress $\sigma_{x x}$ and $\sigma_{y y}$; (c) load-displacement curves of point A with a soft material; and (d) load-displacement curves of point A with a rigid material.

1 Two different fictitious materials, both having the same $G_{\mathrm{f}}\left(\overline{\mathscr{H}}^{\text {large }}=0.4\right.$ and $\left.\overline{\mathscr{H}}^{\text {small }}=0.2\right)$, and different elastic properties (see Figures 8(c) and 8(d)) have been then considered. The

3 rigid material has elastic properties 1000 times larger than the soft one. This precludes, in the former, large elastic strains and displacements to develop at the bulk, unlike in the soft

5 material case. As can be checked in Figure 8(c), the results obtained assuming finite strain or infinitesimal strain kinematics are quite different for the soft material (which allows the plates

7 to undergo large strains and displacements). However, for the rigid material case, Figure 8(d) shows very similar responses for both types of kinematics since large strains do not develop at the bulk and the separation law is made equivalent for both cases through Equation (80). 


\section{NME 607}

These analyses show that, despite the considered kinematics does make a difference in the results, if the regular strains are small the consideration of the fracture energy as a material

3 property makes those results more insensible to the type of kinematics used to model the discontinuous interface $\mathscr{S}$.

5 In Figure 8(c), invariance of the results with respect to the regularization parameter $k$ is

7 also shown through comparison of the results obtained with two different values $\left(k=10^{-3}\right.$ and $\left.10^{-5}\right)$.

\section{CONCLUSIONS}

9 Throughout the previous sections the strong discontinuity approach (SDA) in finite strain settings has been explored. Although the topic had already been tackled in different contexts

$11[8,9]$ here we have extended the results found in infinitesimal strain settings $[17,18,25]$ to the large strain case. As the main result we have shown that the strong discontinuity analysis

13 procedures used there can be extended to the large strain case, by changing the considered strong discontinuity kinematics (7), and the same set of conclusions are achieved, i.e.

15 - Regularization of the softening modulus $\mathscr{H}$ in the continuum (stress-strain) constitutive model (23) induces, via the traction continuity condition (14) and the softening regu-

17 larization condition (39), a projected discrete (traction-displacement jump) constitutive model, (48), at the discontinuity interface (see Figure 5).

19 - This fact requires a particular stress structure to be reached at the discontinuous interface, that is determined by the strong discontinuity conditions, (43).

21 - The variable bandwidth model of Section 3.2 provides a tool to automatically induce those strong discontinuity conditions and to relate them to the fracture process zone

23 concept, classically considered in fracture mechanics [25].

- In addition, the induced discrete constitutive model keep the character (continuum damage) of the original continuum constitutive one and have the feature of being a rigid model (the initial stiffness is infinite).

- As in the infinitesimal strains case, the initiation and propagation of the displacement discontinuity can be here determined via standard procedures supplied by the discontinuous bifurcation analysis for finite strain cases.

- Finally, and as the most distinguishing feature of the presented approach, for practical purposes the complete analysis and simulation can be done in a continuum format, both for the continuous and discontinuous regimes, since the discrete constitutive model is automatically induced from the traction continuity and the softening regularization.

Through the numerical simulations performed in this work, it has been proved its ability 35 to capture strong discontinuities also when large strain kinematics are considered. The main drawback of this type of finite element i.e. the necessity of a global||l|| algorithm to track

37 the discontinuity across the finite element mesh, remains in the large strains context. The global character of this algorithm makes its implementation in typical finite element codes cumbersome, and difficult to deal with multiple crack problems, branching phenomena, etc.

"|||| The global character means that the algorithm cannot be implemented only affecting the one element level (local level) of a finite element code, but at higher levels of the algorithmic structure of the code. 


\section{NME 607}

1 The numerical simulations also corroborate the predictions of the strong discontinuity analyses, i.e. the relevance of the strong discontinuity conditions and the role of the transition

3 (weak discontinuity) regime, and the proposed variable bandwidth model, to make the simulations physically consistent [25] as well as the relevance of the type of kinematics (large or

5 small results) in the obtained results.

\section{APPENDIX A: CONSTITUTIVE TANGENT TENSOR LOCALIZATION} CONDITION, INCREMENTAL INTEGRATION

In this section additional details related to the damage constitutive model of Section 3.5 are

9 presented. First we particularize the damage function (23e) by adopting:

$$
\phi(\boldsymbol{\sigma}, q)=\sqrt{\boldsymbol{\sigma} \cdot \mathbf{c}_{\phi}^{-1} \cdot \boldsymbol{\sigma}}-q ; \quad \mathbf{c}_{\phi}^{-1}=\frac{1}{2 \mu_{\phi}} \mathbf{I}-\frac{\lambda_{\phi}}{2 \mu_{\phi}\left(2 \mu_{\phi}+3 \lambda_{\phi}\right)} \mathbb{1} \otimes \mathbb{1}
$$

11 I being the fourth-order identity tensor. The surface $\phi(\sigma, q)=0$ defines an ellipsoid of revolution in the stress space, where parameters $\mu_{\phi}$ and $\lambda_{\phi}$ governs the ratio among its major and 13 minor axis.

The constitutive tangent tensor associated to this damage function is given by

$$
\begin{aligned}
L_{\overline{\mathbf{v}}} \tau= & \mathbf{c}^{\operatorname{tang}}: \mathbf{d}=\mathbf{c}^{\operatorname{tang}}:\left(\nabla_{\mathbf{x}} \dot{\mathbf{u}}\right)^{S} \\
\mathbf{c}^{\operatorname{tang}}= & \frac{q}{r} \mathbf{c}^{e}+\left(\frac{r q_{, r}-q}{r^{3}}\right)\left[\frac{\theta-2 \kappa \operatorname{tr}(\bar{\tau})}{J^{2}} \bar{\tau} \otimes \bar{\tau}\right. \\
& \left.+\left(\frac{\zeta \operatorname{tr}(\bar{\tau})}{J^{2}}-r^{2}\right) \bar{\tau} \otimes \mathbb{1}+\frac{2 \omega}{J^{2}} \bar{\tau} \otimes \bar{\tau}^{2}\right] \quad \text { if } \dot{r}>0 \\
\mathbf{c}^{\operatorname{tang}}= & \frac{q}{r} \mathbf{c}^{e} \quad \text { if } \dot{r} \leqslant 0
\end{aligned}
$$

15 where $\bar{\tau}=(r / q) \tau=(1-d) \tau$ is the effective Kirchhoff stress, and $\mathbf{c}^{e}$ is the hyperelastic constitutive tensor:

$$
\begin{aligned}
& \mathbf{c}^{e}=\lambda^{*}(\mathbb{1} \otimes \mathbb{1})+2 \mu^{*} \mathbf{I} \\
& \lambda^{*}=\lambda J^{2} ; \quad \mu^{*}=\mu+\frac{\lambda}{2}\left(1-J^{2}\right)
\end{aligned}
$$

17 where $\lambda, \mu$ are the Lame's parameters of the hyperelastic law (Equation (23a)), and the scalar factors $\zeta, \theta$ are:

$$
\begin{aligned}
& \zeta=\lambda^{*} \omega-3 \kappa \lambda^{*}-2 \mu^{*} \kappa ; \quad \theta=2 \mu^{*} \omega \\
& \omega=\frac{1}{2 \mu_{\phi}} ; \quad \kappa=\frac{\lambda_{\phi}}{2 \mu_{\phi}\left(2 \mu_{\phi}+3 \lambda_{\phi}\right)}
\end{aligned}
$$


1

We write the localization tensor $\mathbf{Q}^{L}$ of Section 3.3 as follows:

$$
\mathbf{Q}^{L}=\frac{q}{r} \mathbf{Q}^{e}\left(\mathbb{1}+\left[\frac{q, r}{r q}-\frac{1}{r^{2}}\right] \boldsymbol{\xi} \otimes \boldsymbol{\rho}\right)
$$

$3 \mathbf{Q}^{e}=\mathbf{e}_{n} \cdot \mathbf{c}^{e} \cdot \mathbf{e}_{n}+\left(\mathbf{e}_{n} \cdot \bar{\tau} \cdot \mathbf{e}_{n}\right) \mathbb{1}$ being the acoustic elastic tensor satisfying $\operatorname{det}\left(\mathbf{Q}^{e}\right)>0$, and vectors $\xi$, $\rho$ being given by

$$
\begin{aligned}
& \xi=\left(\mathbf{Q}^{e}\right)^{-1} \cdot \tau \cdot \mathbf{e}_{n} \\
& \boldsymbol{\rho}=\frac{\theta-2 \kappa \operatorname{tr}(\bar{\tau})}{J^{2}} \tau \cdot \mathbf{e}_{n}+\left(\frac{\zeta \operatorname{tr}(\tau)}{J^{2}}-q r\right) \mathbf{e}_{n}+\frac{2 \omega}{J^{2}} \bar{\tau}^{2} \cdot \mathbf{e}_{n}
\end{aligned}
$$

Recalling the term $q_{, r}=\mathscr{H}$, the critical softening modulus $\mathscr{H}^{\text {crit }}$ which makes singular the localization tensor $\mathbf{Q}^{d}\left(\operatorname{det}\left(\mathbf{Q}^{d}\right)=0\right)$, is then determined through the following expression:

$$
\mathscr{H}^{\text {crit }}=\frac{q}{r}\left(1-\frac{r^{2}}{\xi \cdot \rho}\right)
$$

Damage integration algorithm: Box 1 describes the integration algorithm.

Box 1. Damage integration algorithm.

Assume that incremental displacement are given at time $t+\Delta t$.

Then: evaluate the following terms

(i) $\mathbf{F}_{t+\Delta t} ; \mathbf{b}_{t+\Delta t} ; J_{t+\Delta t}$

(ii) $\bar{\tau}_{t+\Delta t}=\lambda \frac{\left(J_{t+\Delta t}^{2}-1\right)}{2} \mathbb{1}+\mu\left(\mathbf{b}_{t+\Delta t}-\mathbb{1}\right)$

(iii) $\phi_{t+\Delta t}^{\text {trial }}=\frac{1}{J_{t+\Delta t}} \frac{q_{t}}{r_{t}} \sqrt{\bar{\tau}_{t+\Delta t} \cdot \mathbf{c}_{\phi}^{-1} \cdot \bar{\tau}_{t+\Delta t}}-q_{t}$

if $\phi_{t+\Delta t}^{\text {trial }} \leqslant 0$ then

there was unloading and the result of the integration step is:

$$
\tau_{t+\Delta t}=\frac{q_{t}}{r_{t}} \bar{\tau}_{t+\Delta t} ; \quad r_{t+\Delta t}=r_{t} ; \quad q_{t+\Delta t}=q_{t}
$$

else if $\phi_{t+\Delta t}^{\text {trial }}>0$ then

there was loading and from the equation $\phi_{t+\Delta t}=0$ it is obtained

$$
r_{t+\Delta t}=\frac{1}{J_{t+\Delta t}} \sqrt{\bar{\tau}_{t+\Delta t} \cdot \mathbf{c}_{\phi}^{-1} \cdot \bar{\tau}_{t+\Delta t}}
$$

which finally determines:

\section{endif}

$$
q_{t+\Delta t}=q_{t}+\mathscr{H}\left(r_{t+\Delta t}-r_{t}\right) ; \quad \tau_{t+\Delta t}=\frac{q_{t+\Delta t}}{r_{t+\Delta t}} \bar{\tau}_{t+\Delta t}
$$


1 This algorithm is slightly modified in the weak discontinuity regime to take into account the bandwidth variation, and hence the softening modulus dependence with $q$.

\section{ACKNOWLEDGEMENTS}

This work has been done with support of the Spanish Ministry of Science and Technology under Grants

5 MAT-2001-3863-C03-03. This support is gratefully acknowledged.

The second author also acknowledges the financial support of the Secretaria de Estado de Educación, Universidades, Investigación y Desarrollo of Spain.

\section{REFERENCES}

1. Planas J, Elices M, Guinea GV. Cohesive cracks versus nonlocal models: closing the gap. International Journal of Fracture 1993; 63:173-187.

9 2. Tvergaard V. Studies of elastic-plastic instabilities. Journal of Applied Mechanics, ASME 1999; 66:3-9.

3. Molinari A, Clifton RJ. Analytical characterization of shear localization in thermoviscoplastic materials. Journal of Applied Mechanics, ASME 1987; 54(December):806-812.

4. Vardoulakis I. Stability and bifurcation in geomechanics: strain localization in granular materials. In Lecture Notes, Univ. Politecnica de Catalunya, October, 1999.

5. de Borst R, Sluys LJ, Muhlhaus HB, Pamin J. Fundamental issues in finite element analyses of localization of deformation. Engineering Computations 1993; 10:99-121.

6. Simo J, Oliver J. A new approach to the analysis and simulation of strong discontinuities. In Fracture and Damage in Quasi-brittle Structures, Bazant ZP et al. (eds). E\&FN Spon, 1994; 25-39.

7. Oliver J. Modeling strong discontinuities in solid mechanics via strain softening constitutive equations. Part 1. fundamentals. International Journal for Numerial Methods in Engineering 1996; 39(21):3575-3600.

8. Armero F, Garikipati K. An analysis of strong discontinuities in multiplicative finite strain plasticity and their relation with the numerical simulation of strain localization in solids. International Journal of Solids and Structures 1996; 33(20-22):2863-2885.

9. Larsson R, Steinman P, Runesson K. Finite element embedded localization band for finite strain plasticity based on a regularized strong discontinuity. Mechanics of Cohesive-frictional Materials 1998; 4:171-194.

10. Larsson R, Runesson K, Sture S. Embedded localization band in undrained soil based on regularized strong discontinuity theory and finite element analysis. International Journal of Solids and Structures 1996; 33(20-22): 3081-3101.

11. Regueiro RA, Borja RI. A finite element model of localized deformation in frictional materials taking a strong discontinuity approach. Finite Elements in Analysis and Design 1999; 33:283-315.

12. Dvorkin EN, Cuitino AM, Gioia G. Finite elements with displacement embedded localization lines insensitive to mesh size and distortions. International Journal for Numerical Methods in Engineering 1990; 30:541-564.

13. Lofti HR, Benson Shing P. Embedded representation of fracture in concrete with mixed finite elements. International Journal for Numerical Methods in Engineering 1995; 38:1307-1325.

14. Oliver J. Modeling strong discontinuities in solid mechanics via strain softening constitutive equations. Part 2: numerical simulation. International Journal for Numerical Methods in Engineering 1996; 39(21):3601-3623.

15. Jirasek M. Finite elements with embedded cracks. Internal report-ecole polytechnique federale de Lausanne, LSC Internal Report 98/01, April 1998.

16. Belytschko T, Moes N, Usui S, Parimi C. Arbitrary discontinuities in finite elements. International Journal for Numerical Methods in Engineering 2001; 50:993-1013.

17. Oliver J. On the discrete constitutive models induced by strong discontinuity kinematics and continuum constitutive equations. International Journal of Solids and Structures 2000; 37:7207-7229.

18. Oliver J, Cervera M, Manzoli O. Strong discontinuities and continuum plasticity models: the strong discontinuity approach. International Journal of Plasticity 1999; 15(3):319-351.

19. Wells GN, Sluys LJ. Application of continuum laws in discontinuity analysis based on a regularized displacement discontinuity. In ECCM'99, European Conference on Computer Mechanics, August 31-September 31999.

20. Garikipati K, Hughes TJR. A study of strain-localization in a multiple scale framework. The one dimensional problem. Computer Methods in Applied Mechanics and Engineering 1998; 159.

21. Armero F. Localized anisotropic damage of brittle materials. In Computational Plasticity. Fundamentals and Applications, Owen DRJ, Onate E (eds). 1997; 635-640.

22. Oliver J. Continuum modelling of strong discontinuities in solid mechanics using damage models. Computational Mechanics 1995; 17(1-2):49-61. 
1 23. Oliver J, Cervera M, Manzoli O. On the use of $\mathrm{J} 2$ plasticity models for the simulation of $2 \mathrm{D}$ strong discontinuities in solids. In Owen DRJ, Onate E, Hinton E (eds). Proceedings of the International Conference on Computational Plasticity, pp. 38-55, CIMNE, Barcelona, Spain, 1997.

24. Oliver J. The strong discontinuity approach: an overview. In Computational Mechanics. New Trends and Applications, Idelsohn S, Oñate E, Dvorkin EN (eds). (WCCM98) Proceedings (CD-ROM) of the IV World Congress on Computational Mechanics, CIMNE, Barcelona, 1998.

7 25. Oliver J. Huespe A, Pulido MDG, Chaves E. From continuum mechanics to fracture mechanics: the strong discontinuity approach. Engineering Fracture Mechanics 2002; 69(2):113-136.

9 26. Bazant ZP, Planas J. Fracture and Size Effect in Concrete and Other Quasibrittle Materials. CRC Press: Boca Raton, FL, 1998.

11 27. Rice JR. The localization of plastic deformation. In Theoretical and Applied Mechanics, Koiter WT (ed.). North-Holland: Amsterdam, 1976; 207-220.

13 28. Willam K. Constitutive models for materials. In Encyclopedia of Physical Science and Technology (3rd edn). Academic Press: New York.

15 29. Bigoni D, Zaccaria D. On strain localization analysis of elastoplastic materials at finite strains. International Journal of Plasticity 1993; 9:21-33.

17 30. Runesson K, Ottosen NS, Peric D. Discontinuous bifurcations of elastic-plastic solutions at plane stress and plane strain. International Journal of Plasticity 1991; 7:99-121.

19 31. Lubliner J. Plasticity Theory. Macmillan Publishing Company: New York, 1990.

32. Zienkiewicz OC, Taylor RL. The Finite Element Method. Butterworth-Heinemann: Oxford, UK, 2000.

21 33. Simo JC, Hughes TJR. Computational Inelasticity. Springer: Berlin, 1998.

34. Jirasek M. Comparative study on finite elements
Mechanics and Engineering 2000; 188:307-330. 